\title{
Detecting Outliers In Multiple Linear Regression
}

م. إيهاب عبد السلام محمود

\author{
By: Ehab A. Mahmood \\ Babylon University / Administration and Economic College
}

\begin{abstract}
:
It is well-known that the existence of outliers in the data will adversely affect the efficiency of estimation and results of the current study. In this paper four methods will be studied to detect outliers for the multiple linear regression model in two cases : first, in real data; and secondly, after adding the outliers to data and the attempt to detect it. The study is conducted for samples with different sizes, and uses three measures for comparing between these methods . These three measures are : the mask, dumping and standard error of the estimate.

\section{اكتشاف القيم الشاذة في الانهدار الفطي المتعدد}

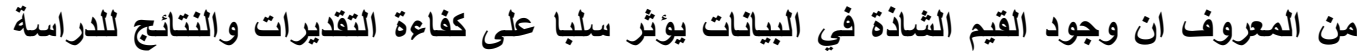

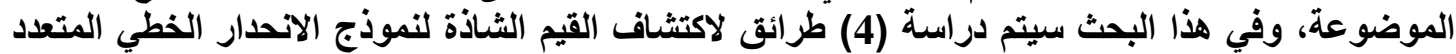

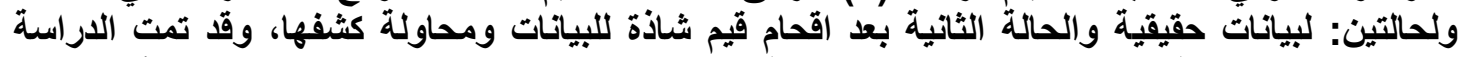

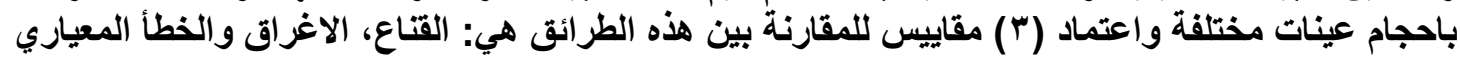




\section{Introduction :}

There are many methods for detecting outliers in linear regression model as:

Elashoff (1972) studied the linear regression model. She illustrated the existing outliers cause the bias in estimator and the high variance . Draper and John (1981) illustrated the benefit of using the Cook Distance.

Pena and Yohai (1999) suggested fast procedure to estimate linear regression parameters in case of existing outliers and how to detect it . Chen (2003) detected outliers in multiple linear regression model. He depended on many robust estimate methods such as (LTS) . Gal ( 2005) presented several methods for the detection of outliers in univariate and multivariate . Karpinski (2007) illustrated in his book the outliers and how to detect them by using several methods .

Mishra (2008) studied several robust and non-robust methods for detecting outliers in multiple linear regression ; he used a Monte Carlo method for comparison between real data and theoretical data .

Asikgil and Erar (2009) tried to determine multiple outliers by using various methods in the presence of masking and swamping effects for the linear regression model .

The multiple linear regression model is as the following equation :

$$
\mathbf{Y}=\mathbf{X} \boldsymbol{\beta}+\boldsymbol{€}
$$

where :

$Y$ : vertical vector $\left(n^{*} 1\right)$ of observed response values.

$X$ : matrix $(n * p)$ of $(p)$ regressors .

$\beta$ : vertical vector $\left(p^{*} 1\right)$ of regression coefficients .

$€$ : vertical vector $(n * 1)$ of error terms .

$\mathrm{n}$ : sample size .

The method of ordinary least squares (OLS) is the most widely used technique to find the best estimates of $(\beta)$ which minimizes the sum of squared distance for actual observations to the regression surface under the assumption $\left(\epsilon \sim \operatorname{NID}\left(0, \sigma^{2} I\right)\right)$; but if the data has outliers the assumption is not satisfied and the estimate dose not minimize the sum of squared distance and will not be optimal . In this case, we must firstly detect outliers and treat them and then apply (OLS) method or we can estimate $(\beta)$ by robust methods of estimate instead of (OLS) method.

Outlier : we can define the outlier as; the observation (or subset of observations) that appear inconsistent (extreme) with the remainder of the data set and has a profound destructive influence on the statistical analysis ; and in linear regression model is not necessarily be extreme (Barnett \& Lewis 1994) .

There are several types of outliers in linear regression :

i. In X-Space : If one or more of the observation values lie far away from the group observations at the $(X)$ axis .

ii. In Y-Space : If one or more of the observation values lie far away from the group observations at the $(Y)$ axis .

iii. In (XY) - Space : If one or more of the observation values lie far away from the group observations at the $(\mathrm{X})$ and $(\mathrm{Y})$ axis. 
Care should be taken in detecting the outlier in set data to prevent masking and swamping problems ; where :

masking ; the unable of the procedure to detect the outliers, swamping; consider the clean observations as outliers (Adnan and others 2003) .

\section{Methods Of Detecting Outliers :}

There are various methods to detect the influential observations in linear regression model. Some of these methods is to detect a single outlier and the other is to detect multiple outliers (single - row diagnostics). The single - row diagnostics can be extended to include subset of observations rather than a single observation (Belsley \& Welsch 1980). In this paper, we will use some of widelyused measures that depend on the single - row diagnostics as the following :

\subsection{Mahalanobis Distance : $\quad$ (McLachlan 1999)-(Mishra 1994)}

Mahalanobis proposed this measure in (1936) to detect contaminated or outlier data points in linear regression model . His measure has played an important role in statistics and data analysis .

The generalized distance can defined as follows :

$$
D_{i}=\sqrt{\left(y_{i}-E\left(y_{i}\right)\right)^{I} S^{-1}\left(y_{i}-E\left(y_{i}\right)\right)}
$$

Where :

$\mathrm{S}$ : covariance matrix . when :

We will reject the null hypothesis; and the observation will be outlier

$D_{i}^{2}>\chi_{(n-p, \alpha)}^{2}$ equation :

In the linear regression model can compute the distance as the following

$$
D_{i}=\sqrt{\left(\hat{y}_{i}-\overline{\hat{y}}\right)^{I} S^{-1}\left(\hat{y}_{i}-\overline{\hat{y}}\right)}
$$

Where :

$\widehat{y}_{i}$ : forecasting value .

$\overline{\hat{y}}$ : forecasting values mean .

We can compute Mahalanobis distance in many statistical packages like SPSS .

\subsection{Cook's Distance : (Cook 1979)}

In (1979) Cook presented a method to detect the influential observation in multiple linear regression which is based on the measure of the distance between $(\hat{\beta})$ and $\left(\hat{\beta}_{i}\right)$ as follows :

$$
D_{i}=\frac{\left(\widehat{\beta}_{i}-\widehat{\beta}\right)^{I} X X\left(\widehat{\beta}_{i}-\widehat{\beta}\right)}{p s^{2}} \leq F_{(p, n-p, 1-\alpha)}
$$


Where :

$\hat{\beta}$ : denotes the least square estimate of $(\beta)$.

$\hat{\beta}_{i}$ : denotes the least squares estimate of $(\beta)$ with the (ith) point deleted.

$S^{2}=\frac{\hat{\epsilon} r \hat{\epsilon}}{n-p}$

If the $\left(D_{i}\right)>F_{(p, n-p, 1-a)}$; then the (ith) single - row is an outliers .

\subsection{Serbert, Montgomery and Rollier Procedure (1998) :}

(Adnan et al, 2003)

They considered a procedure to identify the outliers in multiple linear regression by using the (OLS) method and the single linkage clustering method, where :

The cluster analysis is a method for detecting a natural groupings of items or variables where the items show a high internal homogeneity and low external homogeneity. It includes two groups: hierarchical and non-hierarchical,where the hierarchical method divided into two types :

i. Agglomerative hierarchical method :

It starts with (n) clusters and ends with one cluster which contains all of the data points . (This was conducted by Serbert and et al) .

ii. Divisive hierarchical method :

It starts with one cluster and ends up with (n) clusters with each cluster contains one data point .

The single linkage clustering method : It is a method that depends on the smallest distance between a data point in the first cluster and a point in the second cluster .

Serbert et al method depends on the following steps :

i. Find the standardized predicted values (depending on the OLS) .

ii. Grouping the data set by using the single linkage clustering algorithm (Agglomerative hierarchical method) with Euclidean distance between pairs of standardized predicted values, and this can be graphically shown in the form of a dendogram or tree diagram .

iii. Number of the clusters depend on the height of the cut (stopping rule); which determine as the following equation :

$$
c h=\bar{h}+k s_{h}
$$

Where :

$\bar{h}$ : Average height of the tree.

$k$ : constant .

$s_{h}:$ The standard deviation of the heights .

iv. The clean data set is the largest cluster formed. It includes the median, and the other clusters contains the outliers . 


\subsection{Adnan, Mohamad and Setan Procedure : (Adnan et al 2003)}

Adnan et al (2003) proposed a modified procedure of Serbert et al, where they used the robust fit ( least trimmed of squares (LTS) instead of the ordinary least squares (OLS) fit; then they applied the backward steps of (Serbert and others) procedure, depending on the standardized predicted values or the residual values .

The (LTS) : It is a method of robust regression estimate proposed by Rousseeuw (1984). It minimizes the sum of squared residuals by selecting smallest $(\mathrm{m})$ of residual and $(\mathrm{n}-\mathrm{m})$ residuals are deleted ; and then find the estimators, depending on the $(\mathrm{m})$ observations which satisfy the objective function as the following :

$$
\operatorname{Min}_{\widehat{\beta}} \sum_{i=1}^{m} e_{i}^{2} \quad \text { (Rousseeuw 1984) }
$$

Where :

$$
m=(n / 2)+((p+1) / 2)
$$

The (LTS) has a high breakdown point of up to $(50 \%)$; which is the highest possible value. (Georgiev 2008)

Breakdown point : It is the smallest part of unusual data that can cause to false the estimator .

\section{Application :}

We will apply a aforementioned methods to detecting the outliers : Mahalanobis, Cook, Serbert and Adnan ; and we will use three measures for comparison : masking ; swamping and standard error estimate (which calculate after delete the outliers), we study one of an important disease that infects the people; which is called ( Hepatitis Disease ). It will represent the dependent variable . This disease depends on four tests to detecting it :

i. Glutamate Oxaloacetate Transaminase (G.O.T) .

ii. Glutamate Pyruvate Transaminase (G.P.T) .

iii. Total Serum Bilirubin (T.S.B) .

iv. Alkaline Phosphatase (Alk) .

They will represent the independent variables .

In this paper, we will study several sample sizes, small $(n=25)$; medium $(n=50)$; large $(n=150)$ and for two cases : firstly real values of observations; and secondly, after adding $(\mathbf{1 0 \%})$ of outliers to this observations .

i. $(\mathbf{n}=25)$ :

We will apply the four detecting methods to the real observations which appear in (table 3.1), and to the observations after adding the (3) outliers in the No. of $(5,10,15)$. The results for Mahalanobis and Cook distances are shown in the table (3.2) . 
The dendrograms for Serbert and Adnan for the real observations are shown in figures (3.1),(3.2), respectively; and the dendrograms for the observations after adding the outliers are shown in figures (3.3),(3.4), respectively .

Table (3.1) Independent variables $(n=25)$

\begin{tabular}{|c|c|c|c|c|c|}
\hline No. & Diseased & G.O.T. & G.P.T & Alk & T.S.B \\
\hline 1 & no & Ir & Ir & $7 \mu$ & $7 . \Lambda$ \\
\hline 2 & yes & $\varepsilon$. & $\varepsilon \varepsilon$ & 01. & $V r_{0} 0$ \\
\hline$r$ & no & 17 & $1 \varepsilon$ & 1.1 & 1.9 \\
\hline$\varepsilon$ & yes & $r \ldots$ & мч. & 119 & 10r. \\
\hline 0 & yes & $1 \cdots$ & ror & $1 \wedge r$ & $\Lambda Y_{.} \cdot$ \\
\hline 7 & no & 11 & $r$ & 99 & 7.1 \\
\hline V & yes & Vo & 70 & $r \leq r$ & $9 \wedge$. \\
\hline$\Lambda$ & no & 19 & 11 & $\vee \wedge$ & 0.1 \\
\hline 9 & yes & $1 \ldots$ & rrY & rVq & $r^{\prime} \cdot r_{.}$ \\
\hline 1. & yes & 9. & 90 & 01. & 91.0 \\
\hline 11 & yes & 97 & YVY & YOY & $r \varepsilon r .{ }^{\circ}$ \\
\hline 14 & no & 10 & $1 \varepsilon$ & 7. & $0 . r$ \\
\hline 14 & no & 7 & $\Lambda$ & 9. & 1.. \\
\hline $1 \leq$ & yes & $\Lambda$. & Y I & rrs & $1 \% 7$. \\
\hline 10 & yes & rq & $r \wedge$. & $r \leqslant 7$ & $r \varepsilon . Y$ \\
\hline 17 & no & 11 & 17 & ITV & 1.0 \\
\hline $1 V$ & no & 0 & $V$ & 101 & r.s \\
\hline 11 & yes & $1 \leqslant 1$ & rYA & Y YV & $01 . r$ \\
\hline 19 & yes & VY & $r \wedge$. & $\varepsilon 90$ & 17.9 \\
\hline$r \cdot$ & no & $\varepsilon$ & $\mathrm{V}$ & $\varepsilon$. & 7.1 \\
\hline$Y 1$ & no & IV & 17 & $1 \leq \varepsilon$ & 0.1 \\
\hline$r Y$ & yes & $1 \cdots$ & IVY & $r V$. & $r \varepsilon r .{ }^{\circ}$ \\
\hline$r r$ & yes & Ir & rIr & YYA & ro.. \\
\hline$r \varepsilon$ & yes & $\Lambda$. & $r \varepsilon \varepsilon$ & $r \wedge \Lambda$ & $\leqslant 9.0$ \\
\hline ro & yes & ro & ro & $r \leqslant 0$ & 118.9 \\
\hline
\end{tabular}

Reference : Educational Babylon Hospital for Women and Children 
Table (3.2) Mahalanobis and Cook distance $(n=25)$

\begin{tabular}{|c|c|c|c|c|}
\hline \multirow{2}{*}{ No. } & \multicolumn{2}{|c|}{ Before } & \multicolumn{2}{|c|}{ After } \\
\hline & Mahalanobis & Cook & Mahalanobis & Cook \\
\hline 1 & $11.9910 \mathrm{~V}$ & $\because \cdot r \cdot 7 r$ & Ir...rYr & $\because \cdots \leq 1$ \\
\hline$r$ & $1 . r \vee 1 \leq 1$ & .ヘฯร^ & $1 . r V . O r$ & $\because \cdots \leq \leqslant 9$ \\
\hline$r$ & $1.17 r r$ & ..oror & $1 . \cdots 01 \leq$ &.$\cdots 117$ \\
\hline$\varepsilon$ & $7 . \ldots r \wedge$ & . . & I. HAMY & $\because \cdots r$. \\
\hline 0 & $8.919 \wedge 1$ & $\because \cdot 1 \leqslant V 0$ & $0.1 \leqslant 094$ & •.r^१V \\
\hline 7 & $\cdot V \cdot \wedge r \leq$ & $\because \cdot \varepsilon \leqslant q r$ & $\because V \ldots Y \leq$ &.$\cdots 10$ \\
\hline $\mathrm{V}$ & Y.V५9V. & $\because \cdots 999$ & Y.VマAVY & $\because \cdots v$ \\
\hline$\Lambda$ & $1.1 \leqslant \vee \wedge \wedge$ & $\because \cdot 1109$ & 1.1874. &. .974 \\
\hline 9 & r. .94Yo & $\because \cdots 1 \wedge$ & Y.VYI. E & $\because \cdots r \wedge V$ \\
\hline 1. & $V .2 \wedge 179$ & .990 .9 & V.YVI7I & $. . \leqslant 4 \leqslant 99$ \\
\hline 11 & $. V Y V \leq \Lambda$ & $\because \cdot r \mid \leq 1$ & .01 .07 &.$\cdots 11 \mathrm{~V}$ \\
\hline Ir & Y.VVIVY & $\because \cdots 1 r \varepsilon$ & Y.V $า \leq \varepsilon 1$ & ... \\
\hline ir & 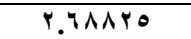 & .1194 & 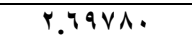 & $\because \cdot 1 \wedge \vee 7$ \\
\hline $1 \leq$ & \&.MINO & $\because \cdot r \leqslant \theta r$ & $\varepsilon . Y r \varepsilon \cdot V$ & $\because \cdot \leqslant 1 Y 4$ \\
\hline 10 & I.r.Ar. & $\because \cdot 10 \wedge 7$ & $1.17 r \wedge 0$ &. $.109 \wedge \mathrm{r}$ \\
\hline 17 & $1 . .7 \wedge 014$ & $. .1 \leq 1 Y 0$ & 1. VRVHO & $\because P V \leq V$ \\
\hline IV & $|r . \leq \wedge \wedge| r$ & $\because \cdots \cdots 1$ & IT.OTYIE & $\because \cdots 194$ \\
\hline 11 & 1.YYYVO & .017 .0 & $1 . Y \leq Y 70$ & $\because \cdots \leq \wedge 1$ \\
\hline 19 & 1.51874 & $.101 \mathrm{~V}$ & $1 . r \mu \mid r$ & $\because \cdots r \wedge q$ \\
\hline$r \cdot$ & Y..9Irr & $\because \cdot 1 \ldots$ & Y.91Y. & ...rr \\
\hline$r 1$ & $1 . \Upsilon 0 \cdot v \wedge$ & $\because \cdot 1 V \cdot 1$ & 1.YOY & $\because \cdots r \leqslant q$ \\
\hline rr & . ATOKr & $\because \cdots \wedge 04$ & $\cdot . \wedge \vee I V \varepsilon$ & $\because \cdots 1 \leq r$ \\
\hline$r r$ & 1.2 .490 & $\because \cdot 1 \leq \wedge 1$ & I.\&.7Y. & $\because \cdots 190$ \\
\hline$Y \leq$ & $9 . \wedge \cdot 1 \vee r$ & $\because \cdot 1 \leq 9$. & $9.7 \vee Y V 7$ & .9 .940 \\
\hline ro & $1 . Y 99 \leq V$ & $\because \cdot 1 \leqslant 09$ & $1 . r \wedge 1.7$ & $\because \cdots r \leqslant V$ \\
\hline
\end{tabular}

Figure (3.1) Serbert before $(n=25)$

\section{Rescaled Distance Cluster Combine}

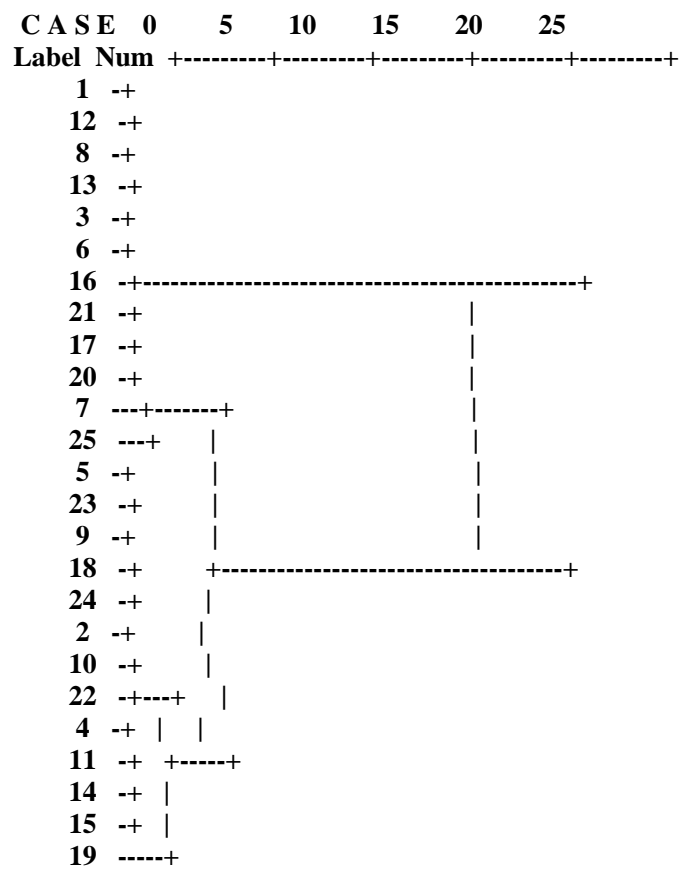


Figure (3.2) Adnan before $(n=25)$

Rescaled Distance Cluster Combine

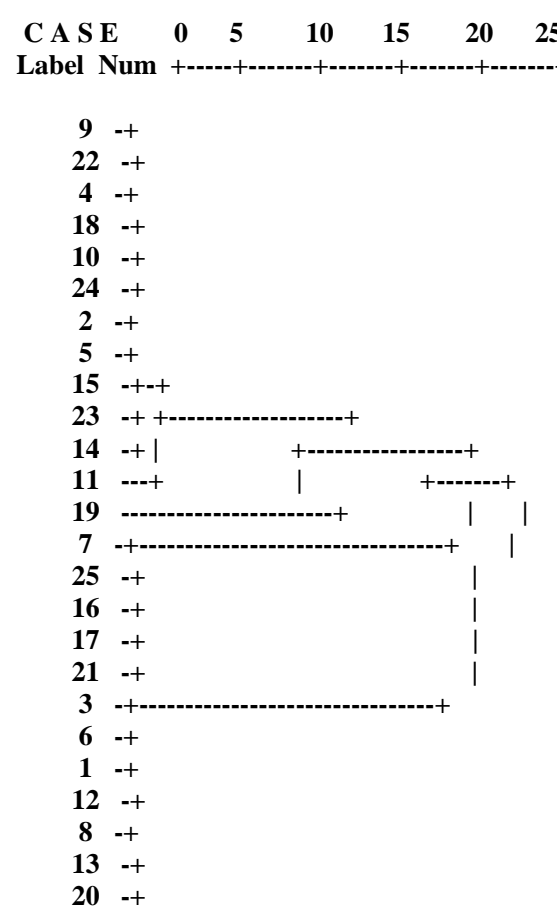

Figure (3.3) Serbert after $(n=25)$

Rescaled Distance Cluster Combine

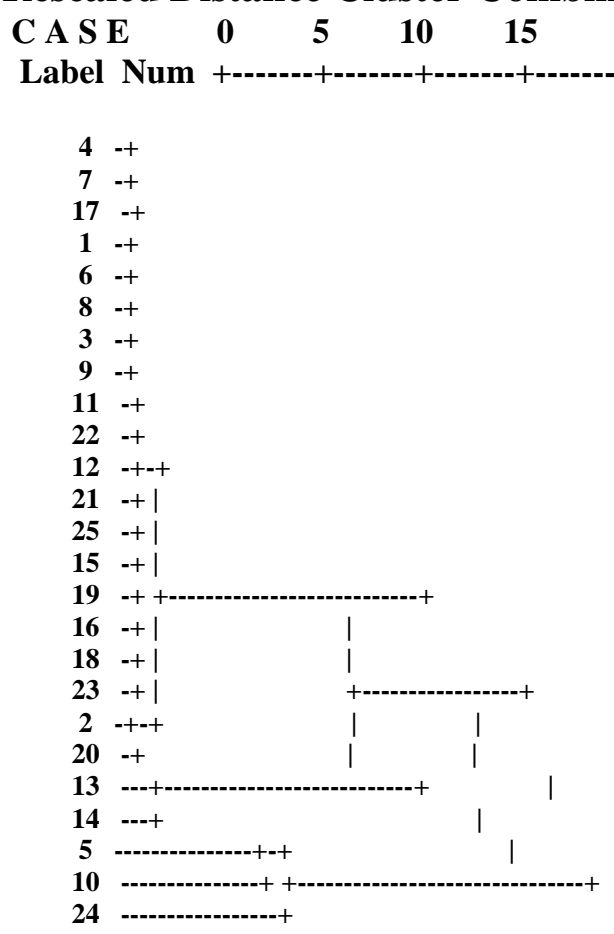


Figure (3.4) Adnan after (n=25)

Rescaled Distance Cluster Combine

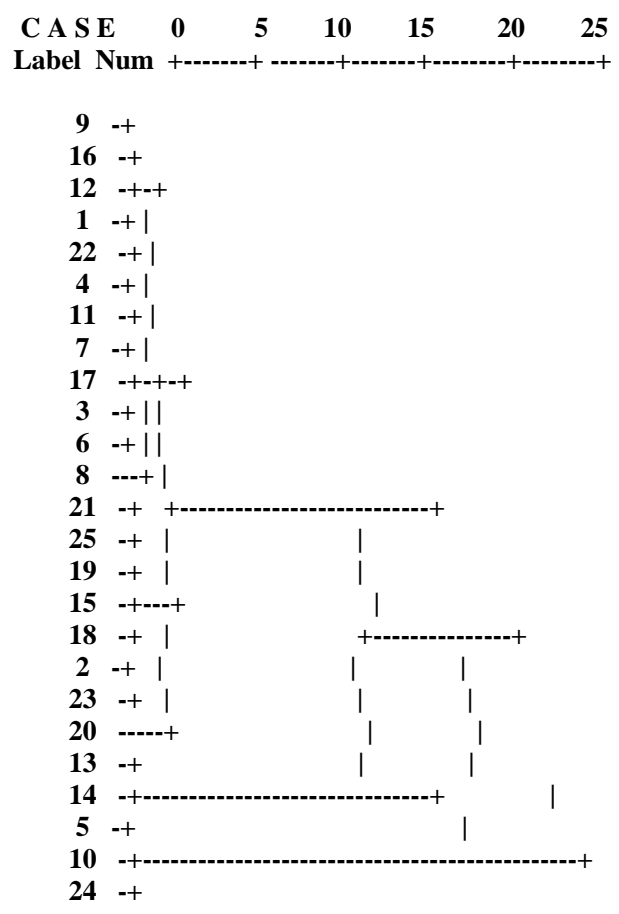

Table (3.3) shows the summary for the methods and contains (7) columns : i. (Case) : Represents before and after adding the outliers .

ii. (Method) : includes (non) i.e without depending on any method for detecting outliers, and the methods of detect .

iii.(ch) : calculate the (ch) value by the equation (2.4) for Serbert and Adnan methods .

iv.(Outliers) : The outliers detected by the methods; where they are all compared with Mahalanobis distance $\left(\chi_{(20,0.05)}^{2}=31.4\right)$, and Cook distance $\left(F_{(5,20,0.05)}=2.71\right)$. Both methods can not detect any outliers in the two cases . Outliers are detected by Serbert and Adnan, depending on (ch) value . Differences are found between them for (before) case, and same results for the (after) case .

v. (Masking) : Mahalanobis and Cook have masking for all the adding outliers ; but Serbert and Adnan have only No. (15) .

vi. (Swamping) : Mahalanobis and Cook did not have swamping; but Serbert and Adnan have the same swamping in the No. $(13,14,24)$.

vii. (Std. Error Est.) : Adnan has (0.09) ; which is less than others for (before) case , and for the (after) case Serbert and Adnan have (1.2) which is less than others . 
Table (3.3) Summary $(n=25)$

\begin{tabular}{|c|c|c|c|c|c|c|}
\hline case & method & ch & outliers & masking & swamping & $\begin{array}{c}\text { Std. } \\
\text { Error } \\
\text { Est. }\end{array}$ \\
\hline \multirow{5}{*}{ before } & none & - & - & - & - & 0.2 \\
\hline & Mah. & - & - & - & - & 0.2 \\
\hline & Cook & - & - & - & - & 0.2 \\
\hline & Serbert & 8.6 & $(16,17,20,21)$ & - & - & 0.2 \\
\hline & Adnan & 11.1 & $\begin{array}{c}(3,7,16,17,19 \\
21,25)\end{array}$ & - & - & 0.09 \\
\hline \multirow{5}{*}{ after } & none & - & - & - & - & 1.58 \\
\hline & Mah. & - & - & $5,10,15$ & - & 1.58 \\
\hline & Cook & - & - & $5,10,15$ & - & 1.58 \\
\hline & Serbert & 9.9 & $(5,10,13,14,24)$ & 15 & $(13,14,24)$ & 1.2 \\
\hline & Adnan & 9.9 & $(5,10,13,14,24)$ & 15 & $(13,14,24)$ & 1.2 \\
\hline
\end{tabular}

ii. $(\mathbf{n}=\mathbf{5 0})$ :

We will apply the detecting methods to the real observations which are shown (table 3.4), and to the observations after adding the (5) outliers in the No. of $(1,10,20,35,45)$. The results for Mahalanobis and Cook distances are shown in the table (3.5); the dendrograms for Serbert and Adnan for the real observations are shown in figures (3.5),(3.6), respectively. The dendrograms for the observations after adding the outliers will are shown in figures (3.7),(3.8), respectively .

Table (3.4) Independent variables $(n=50)$

\begin{tabular}{|c|c|c|c|c|c|}
\hline No. & Diseased & G.O.T. & G.P.T & Alk & T.S.B \\
\hline 1 & yes & 00 & $1 \times 9$ & YrA & $r \leq r . *$ \\
\hline 2 & yes & rq & $\Lambda$. & VY & $r \cdot 0^{\circ}$ \\
\hline 3 & yes & $Y \leq$ & ro & 11. & $1 \% \cdot$ \\
\hline 4 & yes & YY & $r \leqslant \Lambda$ & 191 & $\leqslant \vee . \wedge$ \\
\hline 5 & yes & 0. & 19 & $r v$. & ri.. \\
\hline 6 & yes & TV & $r v$ & $1 \%$ & TY.O \\
\hline 7 & yes & 01 & $r V$. & $r \cdot V$ & $V 1.0$ \\
\hline 8 & no & 0 & $\varepsilon$ & 114 & $0 . \wedge$ \\
\hline 9 & yes & Tr & W. & Yos & $r \leqslant . \cdot$ \\
\hline 10 & yes & $r v$ & YTY & $\leqslant \wedge 1$ & 11.. \\
\hline 11 & yes & $r 1$ & 0 . & 199 & $01 . r$ \\
\hline 12 & yes & 97 & TrY & $r \otimes \Lambda$ & Ar.r \\
\hline 13 & yes & $\vee \wedge$ & וT & $r \leq \Lambda$ & 10.0 \\
\hline 14 & yes & 11. & $\Gamma / \Lambda$ & rol & 4.4 \\
\hline 15 & no & 10 & 11 & 9. & T.s \\
\hline 16 & yes & $1 . \varepsilon$ & MIr & $r \leq \Lambda$ & $r \leq r . \cdot$ \\
\hline 17 & yes & $10 Y$ & M. & 199 & 70.0 \\
\hline 18 & no & 7 & $\Lambda$ & $1 \cdots$ & T.s \\
\hline 19 & no & 9 & $\Lambda$ & qY & 0.1 \\
\hline 20 & no & 0 & 0 & $r q$ & 1.0 \\
\hline 21 & no & 19 & ir & $\wedge \wedge$ & $7 . \wedge$ \\
\hline 22 & yes & $r r$ & 171 & $r \leq r$ & 10.0 \\
\hline 23 & no & IV & 17 & 79 & $0 . r$ \\
\hline 24 & yes & $r v$ & $\Lambda$. & $\leqslant 9$. & 1.7. \\
\hline 25 & no & 9 & 0 & $\Lambda V$ & $\Lambda . r$ \\
\hline 26 & no & 14 & IY & 74 & $7 . \wedge$ \\
\hline 27 & yes & $\varepsilon$ & $\leq \leqslant$ & 01. & $V r .0$ \\
\hline 28 & no & 17 & $1 \leq$ & 1.1 & $\wedge .9$ \\
\hline 29 & yes & $r \ldots$ & 19. & 119 & $10 \%$. \\
\hline 30 & yes & $1 \ldots$ & roy & $I \wedge T$ & $\Lambda r_{.} \cdot$ \\
\hline 31 & no & 11 & $r$. & 99 & $7 . \wedge$ \\
\hline 32 & yes & $\varepsilon$ & ro & $r \leq r$ & $9 \wedge . \cdot$ \\
\hline 33 & no & 19 & 11 & $\vee \wedge$ & 0.1 \\
\hline 34 & yes & $1 \ldots$ & YrY & rvq & $r^{\prime} r_{.} \cdot$ \\
\hline 35 & yes & 9. & 90 & 01. & $71 . \cdot$ \\
\hline
\end{tabular}




\begin{tabular}{|c|c|c|c|c|c|}
\hline 36 & yes & 99 & YVY & YOY & $r \leqslant r_{.} \cdot$ \\
\hline 37 & no & 10 & $1 \leq$ & 7. & $0 . r$ \\
\hline 38 & no & 7 & $\Lambda$ & 9. & ^.. \\
\hline 39 & yes & $\Lambda$. & 117 & Yrs & $1 \% 4$. \\
\hline 40 & yes & rq & $r \wedge$. & $Y \leq Y$ & $r \leqslant . Y$ \\
\hline 41 & no & 11 & 17 & $1 \mathrm{rV}$ & $\Lambda .0$ \\
\hline 42 & no & 0 & $\mathrm{~V}$ & 101 & r.s \\
\hline 43 & yes & $1 \leqslant \Lambda$ & rYA & YTV & $01 . r$ \\
\hline 44 & yes & 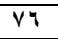 & $r \wedge$. & $\leqslant 90$ & Vฯ. \\
\hline 45 & no & $\varepsilon$ & $\mathrm{V}$ & $\varepsilon$. & $7 . \wedge$ \\
\hline 46 & no & IV & 17 & $1 \leq \leq$ & 0.1 \\
\hline 47 & yes & $1 \ldots$ & IVY & $r V$. & $r \leq r^{*}$ \\
\hline 48 & yes & ITr & rIY & YYA & ro.. \\
\hline 49 & yes & $\Lambda$. & $r \leq \varepsilon$ & $r \wedge \Lambda$ & $\$ 9.0$ \\
\hline 50 & yes & ro & ro & $r \leqslant 0$ & 118.9 \\
\hline
\end{tabular}

Reference : Educational Babylon Hospital for Women and Children .

Table (3.5) Mahalanobis and Cook distance $(\mathbf{n}=50)$

\begin{tabular}{|c|c|c|c|c|}
\hline \multirow{2}{*}{ No. } & \multicolumn{2}{|c|}{ Before } & \multicolumn{2}{|c|}{ After } \\
\hline & Mahalanobis & Cook & Mahalanobis & Cook \\
\hline 1 & $11.00 Y \leq 7$ & $\because \cdots \wedge \cdot \varepsilon$ & YY.Y.YYI & 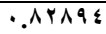 \\
\hline$r$ & 1.577 .0 & $\because .87 .0$ & $1 . .479$. & $\because \cdots \cdots$ \\
\hline$r$ & •.Аएq१V & $\because \leq 0, r$ & $\cdot . \wedge r \wedge Y V$ & $\because \cdots 10$ \\
\hline$\varepsilon$ & $7 . \cdot \leq \vee 9 \wedge$ & $\because \cdot M 9 \wedge \leq$ & 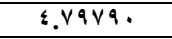 & $\because 119$ \\
\hline 0 & $r . r \leq 1 r$. & $\because|Y| \wedge$ & $r . I Y \leq \leqslant \Lambda$ & ...r. \\
\hline 7 & $\cdot .71 \leq 91$ & $\because .9974$ &. TYYO. & $\because \cdots 7$ \\
\hline $\mathrm{V}$ & Y.1人ระI & $\because \cdots 971$ & Y.OY.Mq & $\because \cdots 7$ \\
\hline$\Lambda$ & $1.17 \mathrm{rVV}$ & $\because \cdots v \leq q$ & $1.11 \leq 19$ & $\because \cdots \vee \leq$ \\
\hline 9 & $\varepsilon . Y O \wedge \vee 0$ & $\because \cdots r \wedge 0$ & $\leqslant . \leqslant 779 \leqslant$ & $\because \cdots 79$ \\
\hline 1. & $\Lambda .0 \cdot \leqslant \Lambda r$ & $\because .0791$ & $1.479 \leq V$ & $\because 9 \leq .41$ \\
\hline 11 & .0 .094 & $\because Y O \wedge 0$ & $.0 \mathrm{OHAS}$ & $\because \ldots \ldots$ \\
\hline $1 Y$ & Y.SVY4A & $\because \cdots 10$ & $Y \leq 1 \leq Y 1$ & $\because \cdots \vee \wedge$ \\
\hline 14 & 5.29111 & $\because \cdots \wedge \leq r$ & r.A0IrA & $\because \ldots 04$ \\
\hline 15 & r..YYY. & ・・7Y & $r . \cdot V Y \cdot V$ & $\because \cdots \leqslant 07$ \\
\hline 10 & $1 . r \cdot r \cdot v$ & $\because \cdots \leqslant r r$ & 1.YOYY & $\because \ldots 09$ \\
\hline 17 & $9.919 \vee 9$ & .0117 & 9.10817 & $\because \cdot 1 \leq V V$ \\
\hline IV & 8.9 .897 & $\because 0009$ & 7.0907 & $\because \cdot 1 \wedge r Y$ \\
\hline 11 & $1.89 \mathrm{rg}$ & $\because \cdots \leq 70$ & 1.11 .19 & $\because \cdots \wedge \wedge$ \\
\hline 19 & $1 . r \cdot \Lambda \cdot r$ & $\because \cdots \leq 11$ & $1 . \mathrm{rrolv}$ & $\because \cdots \wedge r$ \\
\hline$r$. & r.IYOHY & $\because \cdots 1.9$ & r. $\leqslant 9 T \leq \Lambda$ &. $.11 Y Y Y$ \\
\hline$Y 1$ & 1.rYหAV & $\because \cdots \leqslant 7 Y$ & 1.YAYYE & ...Or \\
\hline YY & 1.Y7VI. & $\because \cdots 919$ & $. \wedge 9 \vee / 1$ & $\because \cdots \wedge$ \\
\hline$r Y$ & $1.0 \ldots \leq 7$ & $\because \cdots \leq 4$ & 1.EYYYq & $\because \cdots v 1$ \\
\hline$r \varepsilon$ & V.ร7VัI & $\because \cdots 1 \leq 1$ & V.rvorI & $\because \cdots 10 \mathrm{~V}$ \\
\hline Yo & I.MTY & $\because \cdots r \wedge r$ & $1 . r \wedge 91 Y$ & $\because \cdots 91$ \\
\hline$r 7$ & $1.0 \wedge V Y$. & $\because \cdots r \wedge r$ & $1 . \leqslant \wedge 9.0$ & $\because \cdots 90$ \\
\hline$r V$ & Q.VTVIV & $\because \cdots \cdots$ & 9.997 .1 & $\because \cdots \wedge Y$ \\
\hline$r \wedge$ & 1.99110 & $\because \cdots 0 \wedge 9$ & $1.91 \leqslant 07$ & $\because \cdots O r$ \\
\hline rq & 10.VYTH & $\because \cdot|r| \leq$ & $11.7499 \leq$ & $\because 9490$ \\
\hline$r$. & $\leq .1 \leq 7 \wedge 9$ & $\because \cdots 10 \leq$ & Y.Y.TY & $\cdot \cdots \pi$ \\
\hline$r$ & $1.1 r \leq 40$ & $\because 007$ & $1 . \wedge \wedge \mathrm{VV}$ & $\because \cdots 0$ \\
\hline rr & 1.047 .1 & $\because .+1.1$ & $1.0 \wedge r 17$ & $\because \cdots 11$ \\
\hline$r r$ & $1 . r 941 r$ & $\because \cdots \leq 19$ & $1 . r 47 V 9$ & $\because \cdots 0 v$ \\
\hline$r \varepsilon$ & $r . r . \leqslant 0 q$ & $\because \cdots r r$ & $Y .7 \wedge \wedge \leq 7$ & $\because \cdots r$ \\
\hline ro & $11.994 \% 1$ & $\because \cdot 11 \leqslant 1$ & Q.0TYV. & $\because 0 \wedge r \leqslant r$ \\
\hline$r 4$ & $9.7 Y Y 9 \leq$ & $\because \times \wedge 10$ & $9.4 \cdot 7 Y \leq$ & $\because \cdot 10 \leq V$ \\
\hline$r v$ & $1.7 Y V \leq 7$ & $\because \cdots r \vee \wedge$ & 1.0TrTr & $\because \cdots \wedge V$ \\
\hline$r \Lambda$ & $1 . r 410 r$ & $\because \cdots \leq 17$ & $1 . Y 01 \wedge \mathrm{V}$ & $\because \cdots 1+\varepsilon$ \\
\hline rq & $.770 \leq$ & $\because \cdots 191$ & $\cdot V \cdot 7 r \cdot$ & $\because \cdots r$ \\
\hline$\varepsilon$ & $0.17 \mathrm{HN}$ & $\because \cdots 99 \leq$ & $\varepsilon .9 .711$ & $\because \cdots r$ \\
\hline$\leqslant 1$ & .901 .9 & $\because \cdots \wedge \leq 0$ & $.9 \leq 7 \leq 1$ & $\because \cdots r \leq$ \\
\hline$\varepsilon r$ & $1.10 \mathrm{r} 1 \mathrm{~V}$ & $\because \cdots 9 r \leqslant$ & $1.1 \leqslant 091$ & $\because \cdots 74$ \\
\hline$\varepsilon r$ & $7 . \wedge \leq \leq \leq \leq$ & $\because \cdots \wedge 9$ & 0.11707 & $\because \cdot I V Y \wedge$ \\
\hline
\end{tabular}


$r$.

\begin{tabular}{|c|c|c|c|c|}
\hline$\leqslant \varepsilon$ & $0 . V 9 r \leq Y$ & .0487 & I.VTVIr & ...orl \\
\hline$\leqslant 0$ & $r .074 q$ & $\because \cdots 1 \%$. & $Y . \& \wedge Y \circ Q$ &. .11119 \\
\hline$\leq 7$ & .97001 & $\because \cdots 9 \ldots$ & $\cdot .90 \wedge r Y$ & $\because \cdots r r$ \\
\hline$\leqslant V$ & $1 . . r 19 \leq 0$ & $\because \cdots \leq r \mid$ & $11.91 Y \cdot V$ & $\because \cdots 99 \leq$ \\
\hline$\leqslant 1$ & $0.07 . \wedge V$ & $\because \cdots Y V Y$ & $\varepsilon . Y \leq Y \cdot 1$ & .1117 \\
\hline$\leqslant 9$ & 5.1779 & $\because \cdots q r$ & $\xi . \curlyvee \wedge \otimes \wedge \Delta$ & $\because \cdots 111$ \\
\hline 0. & $Y .1 \leq V Y$. & $\because \cdot Y V \cdot r$ & $r .19 \leq r q$ & $\ldots \cdots$ \\
\hline
\end{tabular}

Figure (3.5) Serbert before $(n=50)$

Rescaled Distance Cluster Combine

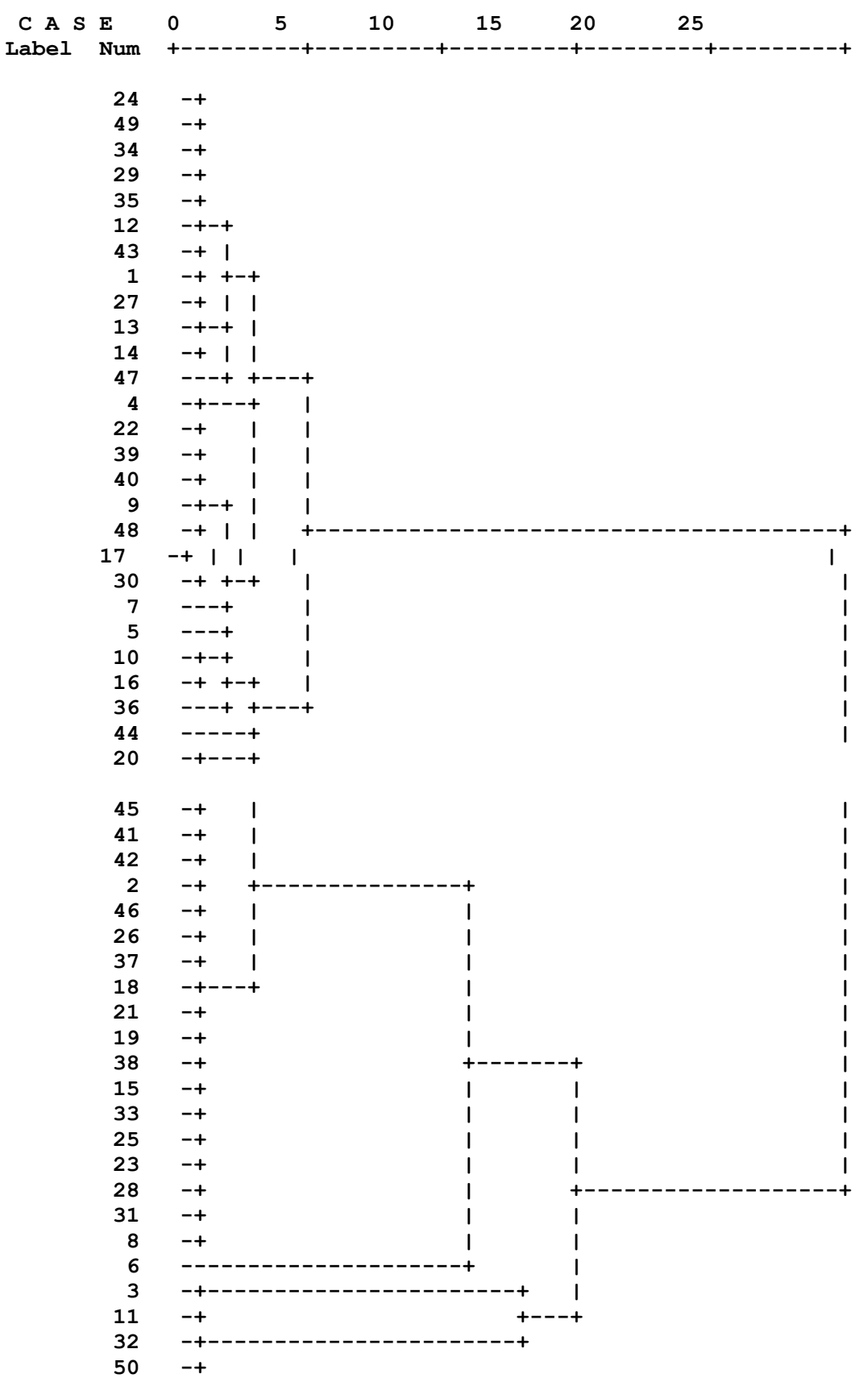


Figure (3.6) Adnan before $(n=50)$

Rescaled Distance Cluster Combine

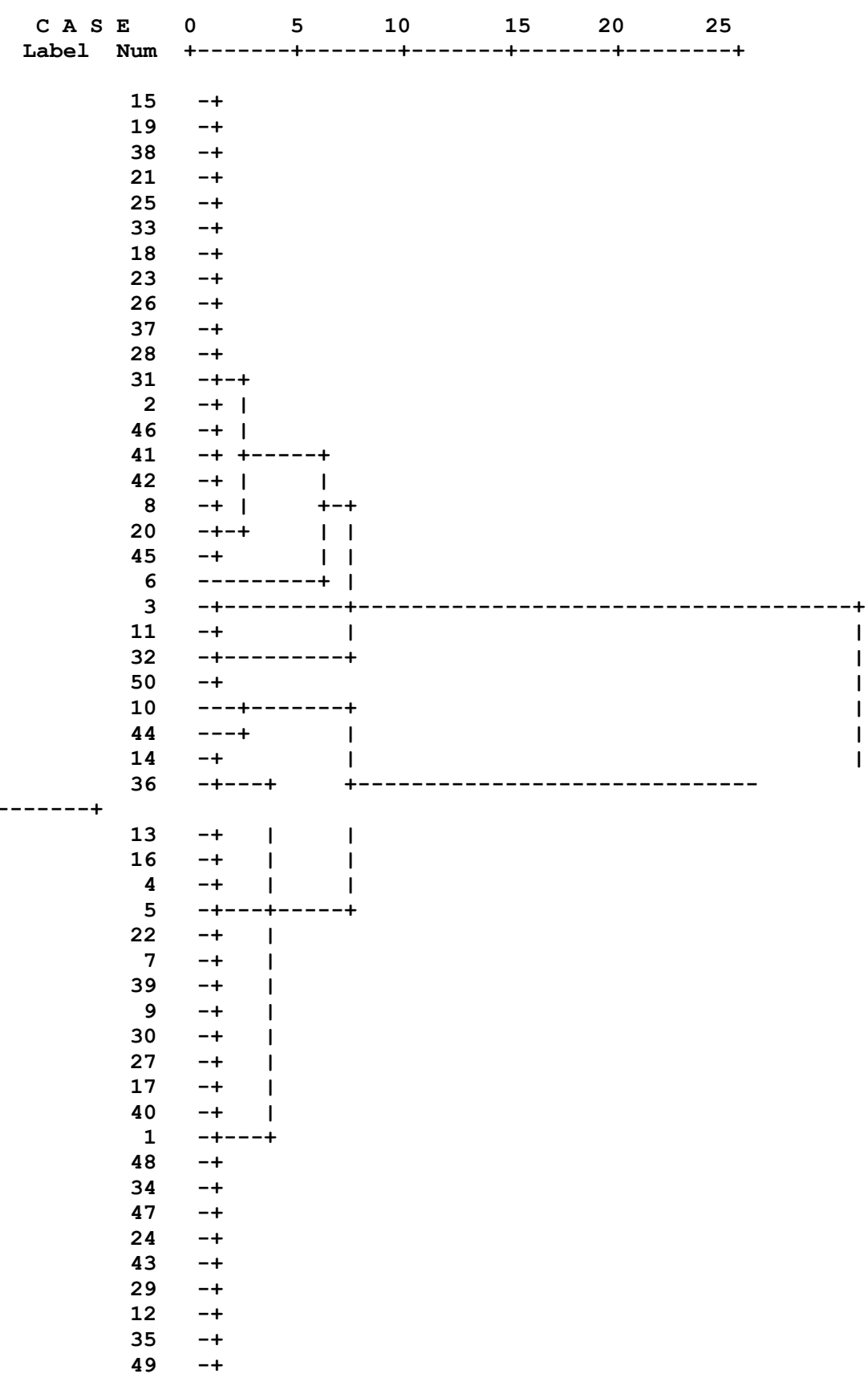


Figure (3.7) Serbert after $(n=50)$

Rescaled Distance Cluster Combine

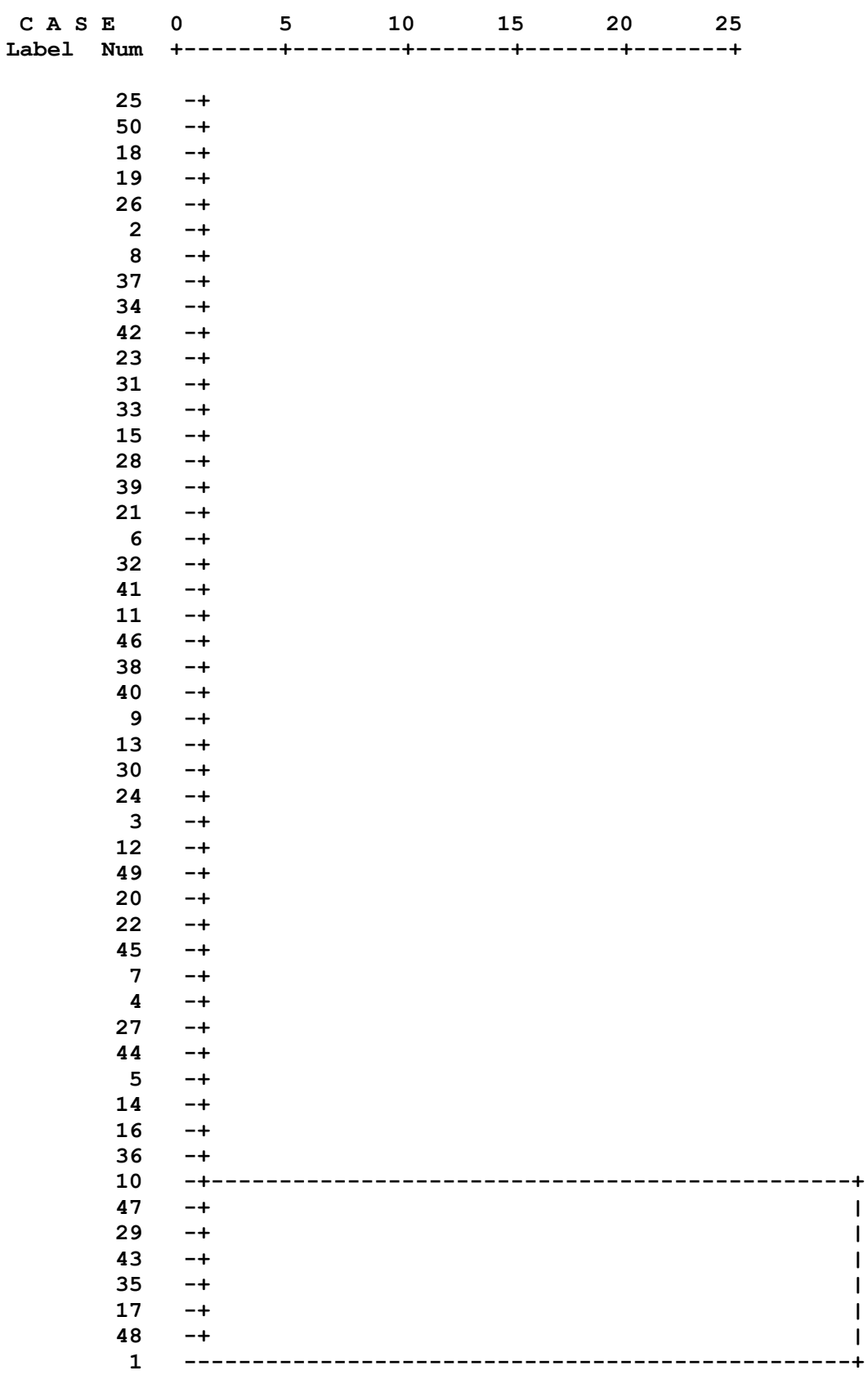


Figure (3.8) Adnan after $(n=50)$

Rescaled Distance Cluster Combine

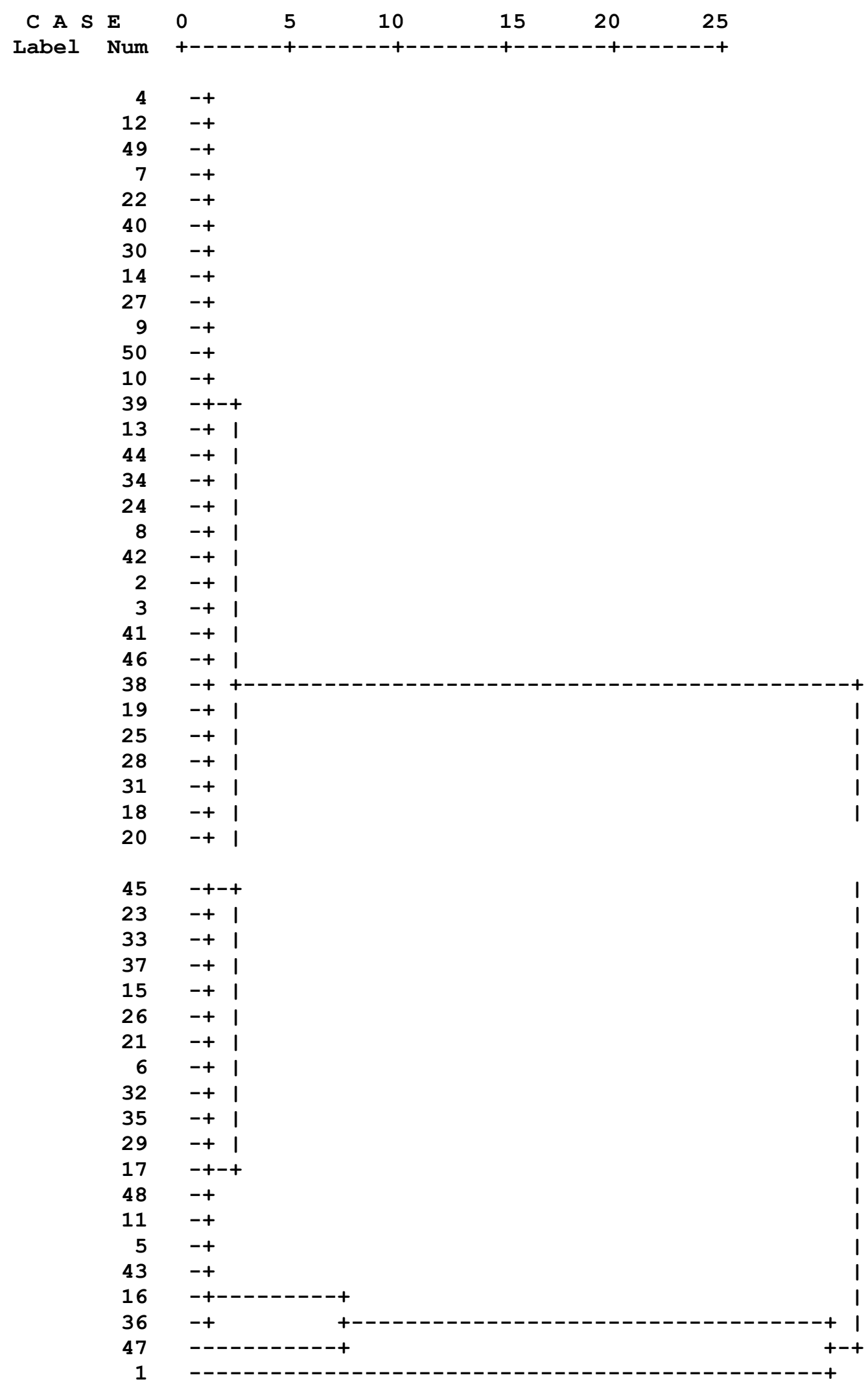


Table (3.6) below shows the summary for the methods where they are all compared with Mahalanobis distance $\left(\chi_{(\{0,0.05)}^{2}={ }^{1} 1.4\right)$, and Cook distance $\left(F_{(5, \leqslant 0,0.05)}=2 . \leqslant r\right)$. Both methods can not detect any outliers in the two cases . Outliers are detected by Serbert and Adnan, depending on (ch) value . Differences are found between them for (before) case, and same results for the (after) case .

Mahalanobis and Cook have masking for all the adding outliers ; but Serbert has only two in the No. $(20,45)$; and Adnan has in the No. $(10,20,35,45)$

Mahalanobis and Cook did not have swamping ; but Serbert has in the No. $(17,29,43,47,48)$; and Adnan has in the No. $(5,11,16,36,43,47,48)$.

Serbert has (0.23),( 2.14) Std. Error Est. before and after cases, respectively ; which are less than others .

Table (3.6) Summary $(n=50)$

\begin{tabular}{|c|c|c|c|c|c|c|}
\hline case & method & ch & outliers & masking & swamping & $\begin{array}{c}\text { Std. Error } \\
\text { Est. }\end{array}$ \\
\hline \multirow{5}{*}{ Before } & none & - & - & - & - & 0.26 \\
\hline & Mah. & - & none & - & - & 0.26 \\
\hline & Cook & - & none & - & - & 0.26 \\
\hline & Serbert & 7.7 & $\begin{array}{c}(3,6,8,11,15,19,21,23,2 \\
5,28,31,32,33,38)\end{array}$ & - & - & 0.23 \\
\hline & Adnan & 6.3 & $\begin{array}{c}(2,3,6,8,11,15,18,19 \\
20,21,23,25,26,28,31 \\
32,33,37,38,41,42,45 \\
46)\end{array}$ & - & - & - \\
\hline \multirow{5}{*}{ After } & none & - & - & - & - & 3.32 \\
\hline & Mah. & - & - & $1,10,20,35,45$ & - & 3.32 \\
\hline & Cook & - & - & $1,10,20,35,45$ & - & 3.32 \\
\hline & Serbert & 11.6 & $\begin{array}{l}(1,10,17,29,35,43,47 \\
48)\end{array}$ & 20,45 & $\begin{array}{c}17,29,43 \\
47,48 \\
\end{array}$ & 2.14 \\
\hline & Adnan & 8.4 & $\begin{array}{l}(1,5,11,16,36,43,47, \\
48)\end{array}$ & $\begin{array}{l}\text { 10,20,35, } \\
45\end{array}$ & $\begin{array}{c}5,11,16,36,43,47 \\
, 48\end{array}$ & 3.46 \\
\hline
\end{tabular}

iii. $\quad(\mathbf{n}=150)$ :

The observations appear in (table 3.7) ; and adding (15) outliers for the No. of $(10,20,35,48,65,70,85,90,100,108,115,125,133,140,148)$.

The results for Mahalanobis and Cook distances are shown in table (3.8) ; the dendrograms for Serbert and Adnan for the real observations are shown in figures (3.9),(3.10), respectively ; and the dendrograms for the observations after adding the outliers are shown in figures (3.11),(3.12), respectively . 
Table (3.7) Independent variables $(n=150)$

\begin{tabular}{|c|c|c|c|c|c|}
\hline No. & Diseased & G.O.T. & G.P.T & Alk & T.S.B \\
\hline 1 & yes & 00 & 149 & rrA & $r \leq Y .{ }^{*}$ \\
\hline$r$ & yes & rq & $\Lambda$. & VY & $r \cdot .0$ \\
\hline$r$ & yes & $Y \leq$ & ro & $1 \wedge$. & YI. \\
\hline$\varepsilon$ & yes & YY & $r \leqslant \Lambda$ & 191 & $\varepsilon V . \wedge$ \\
\hline 0 & yes & 0. & 19 & $r v$. & ri.• \\
\hline 7 & yes & $Y V$ & $r v$ & 17. & rY.o \\
\hline $\mathrm{V}$ & yes & 01 & rV. & $r \cdot V$ & 81.0 \\
\hline$\Lambda$ & no & 0 & $\varepsilon$ & 1179 & 0.1 \\
\hline 9 & yes & 44 & $\mu$. & YOS & $Y \leqslant . \cdot$ \\
\hline 1. & yes & $r v$ & YTY & $\leqslant \wedge 1$ & $\Lambda 1$. \\
\hline 11 & yes & 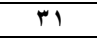 & 0. & 199 & $01 . r$ \\
\hline Ir & yes & 97 & rrr & $r \otimes \Lambda$ & Ar.r \\
\hline 14 & yes & $\mathrm{V \Lambda}$ & rr & $r \leq \Lambda$ & 10.0 \\
\hline $1 \leq$ & yes & 11. & rIN & $r 01$ & 94.4 \\
\hline 10 & no & 10 & 11 & 9. & $r .5$ \\
\hline 17 & yes & $1 \cdot \varepsilon$ & rIr & $r \leq \Lambda$ & $r \leq r . \cdot$ \\
\hline IV & yes & 104 & r. & 199 & 10. \\
\hline 11 & no & 7 & $\Lambda$ & $1 \cdots$ & $r .5$ \\
\hline 19 & no & 9 & $\Lambda$ & QY & 0.1 \\
\hline$r \cdot$ & no & 0 & 0 & rq & 1.0 \\
\hline$r$ & no & 19 & 14 & $\wedge \wedge$ & $7 . \wedge$ \\
\hline YY & yes & $r r$ & 171 & $r \leq r$ & 10.0 \\
\hline$r r$ & no & IV & 17 & 79 & $0 . r$ \\
\hline$r \leq$ & yes & $r v$ & $\Lambda$. & $\leqslant 9$ & 1.7. \\
\hline ro & no & 9 & 0 & $\Lambda V$ & $\Lambda . r$ \\
\hline rq & no & 14 & IY & $7 r$ & $7 . \wedge$ \\
\hline$r V$ & yes & $\varepsilon$ & $\leqslant \leqslant$ & 01. & VT.O \\
\hline$r \wedge$ & no & 17 & $1 \leq$ & 1.1 & 1.9 \\
\hline rq & yes & $r \ldots$ & $r 7$. & 119 & $10 \%$. \\
\hline$r \cdot$ & yes & $1 \ldots$ & roy & $1 \wedge r$ & $\Lambda_{\text {Y.. }}$ \\
\hline$r 1$ & no & 11 & $r \cdot$ & 99 & $7 . \wedge$ \\
\hline rY & yes & $\varepsilon$. & ro & $r \leq r$ & $9 \cdot . \cdot$ \\
\hline$r r$ & no & 19 & 11 & $\vee \wedge$ & 0.1 \\
\hline$r \leq$ & yes & $1 \ldots$ & PrY & $r \vee q$ & $r \cdot r_{.} \cdot$ \\
\hline ro & yes & 9. & 90 & 01. & $71 . \cdot$ \\
\hline ry & yes & 97 & PVY & YOY & $r \leqslant Y .{ }^{\circ}$ \\
\hline$r v$ & no & 10 & $1 \leq$ & 7. & $0 . r$ \\
\hline$r \Lambda$ & no & 7 & $\Lambda$ & 9. & ^." \\
\hline rq & yes & $\Lambda$. & 517 & Yrs & $114 . \cdot$ \\
\hline$\varepsilon$ & yes & $r q$ & $r \wedge$. & $r \leq 7$ & $r \leqslant . r$ \\
\hline$\leqslant 1$ & no & 11 & 17 & IrV & 1.0 \\
\hline$\varepsilon Y$ & no & 0 & $\mathrm{~V}$ & 101 & r.s \\
\hline$\varepsilon r$ & yes & $1 \leq \Lambda$ & $r Y \Lambda$ & YTV & $01 . r$ \\
\hline$\leq \leqslant$ & yes & 87 & $r \wedge$. & $\leqslant 90$ & 87.9 \\
\hline$\leqslant 0$ & no & $\varepsilon$ & $\mathrm{V}$ & \&. & $7 . \wedge$ \\
\hline$\leq 7$ & no & IV & 17 & $1 \leq \leqslant$ & 0.1 \\
\hline$\varepsilon V$ & yes & $1 \ldots$ & IVY & YV. & $r \leqslant r_{.} \cdot$ \\
\hline$\varepsilon \wedge$ & yes & IrT & rIr & $Y Y A$ & ro.s \\
\hline 59 & yes & $\Lambda$. & $r \leq \leq$ & $r \wedge \wedge$ & 29.0 \\
\hline 0. & yes & ro & ro & $Y V T$ & 118.9 \\
\hline 01 & yes & 97 & $r .7$ & $r \wedge V$ & rV. \\
\hline 52 & yes & $r$ & ra & 191 & $r \wedge . \cdot$ \\
\hline 53 & yes & ro & $r$. & $1 \leq V$ & $\varepsilon \wedge . \cdot$ \\
\hline $0 \leq$ & yes & rq & $9 \leq$ & rTV & Yo.. \\
\hline 00 & no & 0 & $\varepsilon$ & 110 & $r .0$ \\
\hline 09 & no & IV & 1. & IYY & $\varepsilon$. \\
\hline$\Delta V$ & no & 11 & $\varepsilon$ & 97 & $r . r$ \\
\hline $0 \wedge$ & no & 0 & 0 & 115 & r.s \\
\hline 09 & yes & 70 & 0. & $r 91$ & 1.9 .8 \\
\hline 7. & yes & ro & 187 & YqV & $V V . \cdot$ \\
\hline 71 & no & 11. & $\varepsilon$ & $1 \ldots$ & $r . r$ \\
\hline IY & no & 7 & 9 & $11 \mathrm{~V}$ & $\Lambda .0$ \\
\hline 74 & yes & 0. & $\leqslant 1$ & 197 & $r \cdot r_{.}$ \\
\hline $7 \leq$ & yes & $\leqslant 1$ & $\leqslant \wedge$ & 114 & $r \cdot r_{.}$ \\
\hline
\end{tabular}




\begin{tabular}{|c|c|c|c|c|c|}
\hline 70 & yes & V. & $\wedge \wedge$ & $9 \ldots$ & $9 .$. \\
\hline 79 & no & $1 T$ & $\varepsilon$ & IrV & 1.0 \\
\hline $7 V$ & yes & IYE & 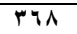 & 191 & Ar.P \\
\hline 71 & yes & 01 & Y Y & 119 & $1 . v_{.} \cdot$ \\
\hline 79 & yes & rq & Yq & 10. & $0 \wedge . \cdot$ \\
\hline v. & no & 9 & $\mathrm{~V}$ & $9 \leq$ & $\Lambda . r$ \\
\hline$v_{1}$ & yes & ro & $r \varepsilon$ & OYO & $9 \wedge . \cdot$ \\
\hline$V Y$ & yes & $V \leqslant$ & Y01 & $01 \mathrm{~V}$ & Y.0.0 \\
\hline$V r$ & yes & 0. & ro. & $\leqslant 97$ & YYO.V \\
\hline$V \leq$ & yes & $1 Y 1$ & YAY & $\varepsilon .0$ & $\Lambda V_{0} \bullet$ \\
\hline vo & no & $\Lambda$ & $\Lambda$ & $9 \leq$ & $\because 0$ \\
\hline VI & yes & Yo. & ro. & 597 & $r \cdot{ }^{\prime} \cdot{ }^{\prime}$ \\
\hline$V V$ & yes & YYE & 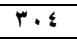 & Yq1 & Y.O. $r$ \\
\hline$V \wedge$ & no & 9 & $\Lambda$ & $\Lambda 1$ &. .5 \\
\hline 89 & no & 11 & $\mathrm{~V}$ & 79 & $\cdot . \wedge$ \\
\hline$\Lambda$. & yes & \& & 1149 & $r . q$ & $1 Y . V$ \\
\hline$\Lambda 1$ & no & $\Lambda$ & 17 & $11 \leq$ & V.V \\
\hline$\Lambda r$ & no & ro & 9 & 00 & .0 \\
\hline 83 & no & $\Lambda$ & 1. & 109 & .9 \\
\hline$\Lambda \varepsilon$ & no & $r V$ & 9 & $1 Y 4$ & $\cdot .9$ \\
\hline 10 & yes & $1 \leq \leqslant$ & $1 \wedge \varepsilon$ & 1.0 & $1 \% .^{\prime}$ \\
\hline 17 & no & 10 & $\varepsilon$ & ITY & 0.9 \\
\hline$\Lambda V$ & no & 9 & 9 & 1.0 & 1.0 \\
\hline$\Lambda \wedge$ & yes & $1 \% 0$ & $Y \leq \cdot$ & $r \wedge$. & $01 . r$ \\
\hline 19 & no & $\Lambda$ & Ir & IYr & 7.1 \\
\hline 9. & yes & $1 Y \wedge$ & $\leqslant \varepsilon$ & r.. & rq. \\
\hline 91 & yes & $1 \leqslant \Lambda$ & ro & 119 & $r \cdot .0$ \\
\hline qr & no & 1. & $1 \leq$ & $11 \leq$ & $114 .$. \\
\hline 94 & yes & $r \varepsilon$ & $r \leqslant$ & $\varepsilon r$ & $1 \wedge .1$ \\
\hline $9 \leq$ & yes & Yo. & $\{Y \wedge$ & $Y \leq q$ & YV.r \\
\hline 90 & yes & $11 \varepsilon$ & YYE & YYY & $\leqslant \Lambda .0$ \\
\hline 97 & no & IV & $\Lambda$ & $\Delta \wedge$ & $0 . Y$ \\
\hline $9 V$ & no & 10 & 10 & $\vee \wedge$ & $0 . r$ \\
\hline 91 & no & 10 & $\varepsilon$ & $1 \%$ & r.0 \\
\hline 99 & yes & 0. & 187 & rq1 & $r \wedge .0$ \\
\hline $1 \ldots$ & no & 0 & 0 & 1.0 & r.o \\
\hline 1.1 & no & IV & $V \leq$ & 94 & $\Lambda .7$ \\
\hline $1 . r$ & no & 10 & 0 & 199 & 0.1 \\
\hline $1 . r$ & no & 11 & 19 & 1.0 & $r . \wedge$ \\
\hline 1.5 & no & 14 & $\mathrm{~V}$ & $1 \%$ & 1.0 \\
\hline 1.0 & no & $\Lambda$ & 9 & $\Lambda \leq$ & 1.0 \\
\hline 1.9 & no & 19 & 0 & $1 . r$ & 0.1 \\
\hline $1 . v$ & no & 1. & $r \cdot$ & $11 \mathrm{~V}$ & 1.0 \\
\hline 1.1 & yes & 7. & $V Y$ & 97 & PY.O \\
\hline 1.9 & yes & $\Lambda r$ & Irr & $11 Y$ & r^.V \\
\hline 11. & yes & $\leqslant 1$ & $V Y$ & 1.0 & $19 . r$ \\
\hline 111 & yes & $1 . \varepsilon$ & $r v$. & $r \ldots$ & $\vee 1 . \wedge$ \\
\hline $11 \%$ & no & 10 & 0 & $1 \%$ & 1.0 \\
\hline $11 \pi$ & no & $\Lambda$ & 1. & 149 & 0.1 \\
\hline $11 \leq$ & no & 9 & $\varepsilon$ & $1 \leq$ & 10.5 \\
\hline 110 & no & 7 & 0 & VY & $1 \leq . r$ \\
\hline 117 & no & 14 & 11 & Ir. & $11 .$. \\
\hline $11 \mathrm{~V}$ & yes & $1 \%$ & rrs & Yo. & 01.0 \\
\hline 111 & yes & 71 & $\Lambda \wedge$ & YY & $r \cdot .1$ \\
\hline 119 & No & IV & 11 & 9. & $0 . r$ \\
\hline Ir. & yes & 110 & Y0Y & $\varepsilon Y$. & $r \wedge . \wedge$ \\
\hline $1 Y 1$ & no & $\Lambda$ & $\Lambda$ & IYr & $9 . \wedge$ \\
\hline IYY & no & 0 & $1 r$ & $V Y$ & $1 \% . r$ \\
\hline Irr & yes & $11 \%$ & $\mu \cdot \varepsilon$ & roq & Yo.0 \\
\hline $1 Y \varepsilon$ & yes & $V$. & MIr & ry. & $\leqslant 0.0$ \\
\hline 1Yo & yes & $\varepsilon V$ & ror & YVq & $18 \cdot .0$ \\
\hline $1 \% 9$ & no & $\Lambda$ & $\Lambda$ & 01 & $r .0$ \\
\hline $1 Y V$ & yes & $\leqslant 1$ & $\$ 17$ & YrA & YYY." \\
\hline IYA & no & 7. & Y & YqA & 1.0 \\
\hline 149 & no & 0 & 0 & 149 & 1.0 \\
\hline
\end{tabular}




\begin{tabular}{|c|c|c|c|c|c|}
\hline $1 \%$ & yes & $\Lambda 0$ & $\varepsilon Y \varepsilon$ & $r q$. & $11 V_{.}$ \\
\hline$|r|$ & yes & ro & Ir. & rq. & $\mu \wedge .0$ \\
\hline ITr & no & 1. & 11 & Ir. & 1.0 \\
\hline Irr & yes & ro & $\Lambda \wedge$ & 19. & $\mu \wedge .0$ \\
\hline $1 \pi \varepsilon$ & yes & $1 . \varepsilon$ & $\varepsilon \ldots$ & rl. & M.^ \\
\hline 110 & yes & $1 \% 0$ & $\varepsilon r Y$ & $r \cdot V$ & or.o \\
\hline 1147 & yes & $r r$ & rrA & YY & 11.0 \\
\hline $1 \% \mathrm{~V}$ & no & $\varepsilon$ & $\varepsilon$ & 9. & $\Lambda .0$ \\
\hline $1 \mathrm{r \Lambda}$ & no & 19 & 9 & 10. & $1 . . r$ \\
\hline 1199 & no & 1. & 1. & 0 . & Ir.r \\
\hline $1 \leqslant$ & no & $\Lambda$ & 7 & 10 & 17.7 \\
\hline $1 \leqslant 1$ & no & 9 & 0 & 77 & $7 . \wedge$ \\
\hline $1 \leqslant Y$ & no & $\Lambda$ & $V$ & $1 . r$ & 0.1 \\
\hline $1 \leqslant r$ & yes & ro & $r \varepsilon$ & $1 \%$. & Y9.1 \\
\hline $1 \leq \varepsilon$ & no & 11 & 0 & 9. & 0.1 \\
\hline $1 \leqslant 0$ & yes & $\Lambda$. & YMY & YYo & $77 . r$ \\
\hline $1 \leq 7$ & no & $\Lambda$ & 9 & 111 & $7 . \wedge$ \\
\hline $1 \leqslant V$ & yes & ro & $r . \xi$ & 10. & Yq. \\
\hline $1 \leqslant \Lambda$ & yes & Or & $r$. & 9. & or.. \\
\hline $1 \leqslant 9$ & yes & $1 \ldots$ & $r \wedge$. & $\{19$ & 11.9 \\
\hline 10. & yes & $\varepsilon V$ & $r \ldots$ & $\leqslant r$. & $r \cdot .0$ \\
\hline
\end{tabular}

Reference : Educational Babylon Hospital for Women and Children .

Table (3.8) Mahalanobis and Cook distance $(n=150)$

\begin{tabular}{|c|c|c|c|c|}
\hline \multirow{2}{*}{ No. } & \multicolumn{2}{|c|}{ Before } & \multicolumn{2}{|c|}{ After } \\
\hline & Mahalanobis & Cook & Mahalanobis & Cook \\
\hline 1 & 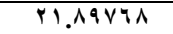 & $\because \cdots 1 \cdot 7$ & IV.Y.VAs & $\ldots \ldots$ \\
\hline$r$ & $1 . \cdots 974$ &. .17 .9 & .9741. & $\ldots \cdots 9$ \\
\hline$r$ & .00419 & $.1 \cdot 71$ & $.0 V Y r V$ & $\ldots \cdots r$ \\
\hline$\varepsilon$ & $\leqslant .01 Y .9$ &. .1 .19 & \&.rrqIr & $\because \cdots+1$ \\
\hline 0 & $r . r_{100}$ &.$\cdots 7.9$ & $r .0701$. & $\ldots \cdots$ \\
\hline 7 & $. . M 0 . Y 4$ & $\because \cdots \wedge 9 \wedge$ & . $Y Y Y \leq V$ & $\because \cdots r$ \\
\hline $\mathrm{V}$ & 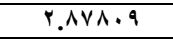 & $\because \cdots+7$ & $r .17 r \leq r$ & $\because \cdots \cdot$ \\
\hline$\Lambda$ & $\cdot . \wedge \wedge \wedge \neg 0$ & $\because 119$ & $\cdot . \Lambda 1 \cdot I V$ & $\because \cdots 1 r$ \\
\hline 9 & $5.0 .91 Y$ & $\because \cdots r q$ & ๕.V।9^r & $\ldots \ldots 9$ \\
\hline $1 \cdot$ & $V .0 \leq 1 Y T$ & $\because \cdots 91$ & YI.VTEYA & $.0 . M 4 \leq$ \\
\hline 11 &. $.1 \wedge 917$ & $\because \cdots \neg \wedge V$ & . \& YYq. & $\because \cdots+1$ \\
\hline $1 Y$ & 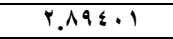 & $\because \cdots \cdots$ & r.rฯ人VI & $\because \cdots 17$ \\
\hline $1 T$ & $r .0 . v 1 r$ & $\because \cdots \leq 9$ & $\varepsilon . .1191$ & $\because \cdots+1$ \\
\hline $1 \leq$ & Y.909V7 & $\because \cdots 7$ & r.rrqin & $\because \cdots 11$ \\
\hline 10 & $\cdot . \wedge \wedge \neg \leqslant \leq$ & $\because \cdots \wedge 0$ &..$\vee \wedge .79$ & $\because \cdots v$ \\
\hline 17 & $I \Lambda . V V \cdot Y \leq$ &.$+4 \cdot 9$ & $10.1 \leq r . \leqslant$ & $\ldots \cdots 1 \leq$ \\
\hline $1 V$ & $\bullet . V \odot \vee \wedge r$ & $\because \cdots \cdots$ & r.7V. $\leq 1$ & $\because \cdots \wedge V$ \\
\hline 11 & $.94 \vee 99$ & $\because \cdots \wedge$ &.$\wedge 0119$ & $\because \cdots 1$ \\
\hline 19 & $.940 \leq 7$ & $\because \cdots v V$ & . ATVVY & $\because \cdots \wedge$ \\
\hline$r \cdot$ & 1.09710 & 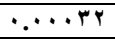 & Y0.TV00s & $\cdot \% 4 \wedge 9$. \\
\hline$Y 1$ & $\cdot . \wedge$ ४ & $\because \cdots 90$ & . VRro. & $\because \cdots 7$ \\
\hline YY & $1.174 \leq 1$ & $. \cdots+01$ & $1.1 \leq 17 \pi$ & $\ldots \ldots$ \\
\hline Yr & $1 . . \leq|Y|$ & $\because \cdots \vee q$ &. $.91 Y K r$ & 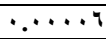 \\
\hline$Y \leq$ & $\vee . \wedge \wedge \cdot \wedge !$ & $\because \cdots 19 r$ & $\Lambda .91 r \leq V$ & $\because \cdots r q$ \\
\hline ro & $.90 \mathrm{Vqr}$ & $\because \cdots v r$ & •.Атル & $\because \cdots \wedge$ \\
\hline YY & $1.1 \mathrm{H} . \mathrm{Ar}$ & $\because \cdots 70$ & $1.114 \mathrm{~V}$ & $\because \cdots$ \\
\hline YV & 9.79911 & ...0YI & $11.1 \times 90$. & $\because \cdots \leq r$ \\
\hline$r \wedge$ & . VYYVE & $\because \cdots 11$ & .74719 & $\because \cdots v$ \\
\hline rq & $1 Y .99100$ & $\because \cdot 1 \cdot v \leq$ & ᄀ.AT. II & . . r r \\
\hline$r \cdot$ & $5 . v 9.1 r$ & ...rr & $0 . Y \vee \wedge / \tau$ & $\because \cdots \leq V$ \\
\hline$r 1$ & $\cdot . V \leq \Lambda \backslash V$ & $\because \cdots 11$ & $. .70 . \mathrm{r}$ & $\because \cdots$ \\
\hline rY & $1.749 \leq \leq$ & $\because \cdots q r r$ & 1. & $\because \cdots+1$ \\
\hline$r r$ & $\cdot .9 \leq \ldots$ & $\because \cdots 91$ & $\cdot \Lambda \cdot v 01$ & $\because \cdots \cdot 0$ \\
\hline$r \leq$ & צ.TYIVI & $\because \cdots r$ & $r . V \vee 0 \leq q$ & $\because \cdots \wedge$ \\
\hline ro & 9.110 .0 & ...r. & A.r. rVO &. .19 .11 \\
\hline
\end{tabular}




\begin{tabular}{|c|c|c|c|c|}
\hline$r q$ & $\mid \wedge . \vee \wedge \leq \neg \wedge$ & $\because .1917$ & $1 \leq .95919$ & $\ldots \cdots v$ \\
\hline$r V$ & $1.10 \mathrm{VVA}$ & $\because \cdots 7 V$ & 1.*KRH & $\because \cdots$ \\
\hline$\mu \wedge$ & $\cdot . q V \leq \leq r$ & $\ldots \cdots v 0$ & $\cdot \wedge \wedge \leq q \wedge$ & $\ldots \ldots 9$ \\
\hline rq & $1.079 \leq$. & $\because \cdots v 0$ & $1.210 \% 1$ & 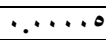 \\
\hline$\varepsilon$ & 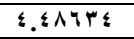 & $\because \cdots \leq 7$. & $\varepsilon . r \wedge \otimes \vee r$ & $\because \cdots r$ \\
\hline$\leqslant 1$ & . . Y $9 \leq \leq \leq$ & $\because \cdots 1 \leq 9$ & .0 OVM & $\because \cdots \wedge$ \\
\hline$\leqslant Y$ & $.9 Y 74 \leq$ & $\because \cdots 1 \leq 7$ & $\cdot . \wedge \leq 79 \mathrm{~V}$ & $\because \cdots 1 \leq$ \\
\hline$\leqslant r$ & $0.1 \% \wedge \wedge 7$ & $\because \cdots r V$ & r.AVYYI & . . . \\
\hline$\leqslant \leqslant$ & $0 . V T r 07$ & .NT07 & $7.54 Y 09$ & $\because \cdots 0$ \\
\hline$\leqslant 0$ & 1.000 .9 & ...r & $1 . \leqslant 7 \leqslant Y V$ & $\because \cdots \wedge$ \\
\hline$\leqslant 7$ & ・.รทท & $\because \cdots 107$ & $.71 \wedge 0 \leq$ & $\because \cdots 9$ \\
\hline$\leqslant V$ & $r \cdot .19 r \leq 9$ & $\because \cdots 7 r \leq$ & $10.7 r Y \wedge 9$ & $\because \cdots 1$ \\
\hline$\leqslant \wedge$ & $\varepsilon .7 \leqslant Y \leqslant V$ & ....rr & $r \wedge . V \leq 191$ & ד \\
\hline$\leqslant 9$ & E.rYTHI & $\because \cdots \cdots 1$ & ¿.VAIrr & ...1. \\
\hline 0. & r.q।rq. & $\because \cdot 1 \cdot 7$ & $Y .9 \leq 99 \mathrm{~V}$ & ..... \\
\hline 01 & $Y .9 \leq \wedge \Lambda \leq$ & $\because \cdots 1 \leq$ & $r .70 \leq 9 r$ & $\because \cdots 71$ \\
\hline 52 & 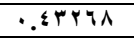 & $\because \cdots \wedge \leq$ & \&.VAITr & $\because \cdots 1$ \\
\hline 53 & . $7 \leqslant$ T & $\because \cdot 11 \vee 0$ & $.0 r 90 r$ & $\because \cdots r$ \\
\hline $0 \leq$ & r.YOVV7 & $\because \wedge \wedge$. & $T . \leqslant 01 \leq \varepsilon$ & $\because \cdots r$ \\
\hline 00 & .9174. & $\because \cdots 91$ & •.А५৭४^ & $\because \cdots 11$ \\
\hline 07 & $. V r \leq V 0$ & $\because \cdots 119$ & $.7040 \leq$ & $\because \cdots \wedge$ \\
\hline$\Delta V$ & $\cdot .9 \cdot \Lambda \leq V$ & $\because \cdots \vee \wedge$ & $\cdot . \wedge 10 Y$. & $\because \cdots \wedge$ \\
\hline $0 \wedge$ & $.9104 \leq$ & $\because \cdots 9$ & •.АYАY & $\because \cdots 11$ \\
\hline 09 & $0 . \leqslant V \leqslant \cdot 1$ & $\because \cdots \leq 74$ & 7. rYqाr & $\because \cdots \cdots$ \\
\hline 7. & Y.10YVA & $\because \cdots+9$ & $1.9 Y \wedge 0 Y$ & $\because \cdots+1$ \\
\hline 71 & N.VM.YI & $\because$ l110r & E.OKY. T & $\because \cdots \leq$ \\
\hline 74 & $\cdot \wedge \leq r Y q$ & $\because \cdots 1 \cdot \leq$ & .07119 & $\because \cdots 11$ \\
\hline 94 & V.VI. II & $\because \cdot r \ldots V$ & 0.91991 & $\because \cdots \leq$ \\
\hline $7 \leq$ & $V . V 0700$ & $\because \cdot Y 1 \wedge q$ & $0.9 \vee 94$. & $\because \cdots \leq$ \\
\hline 70 & $\varepsilon \cdot . \wedge \cdot 1 \cdot q$ & $\cdot . \wedge \cdot \wedge \vee$ & $1.7794 \leq$ & $\because .5 .99$ \\
\hline 77 &..$V 0941$ & $\because \cdots 11 \wedge$ & $. .79 \leq \leqslant r$ & $\because \cdots 9$ \\
\hline $7 V$ & $0 .+\leq 0 \vee \leqslant$ & $\because \cdots v$ & $. .79 \leq \leqslant r$ & $\because \cdots 9$ \\
\hline 71 & 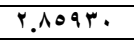 & $\because \cdots 1 \wedge 9$ & r.PIYY & $\because \cdots r$ \\
\hline 79 & $\cdot . \vee \cdot 7 \vee \wedge$ & $\because \cdot 1111$ & $.009 \wedge 4$ & $\because \cdots r$ \\
\hline$V \cdot$ &. .9 .194 & $\because \cdots \wedge 1$ & $11 . \leq r 9 \leq \leq$ & $\cdot . \leqslant r q \wedge r$ \\
\hline VI & $11 . r \wedge . \wedge \leq$ & $\because \cdots 000$ & 19.0111. & $\because \cdots 97$ \\
\hline VY & $\Lambda . \leqslant \Lambda .9 \vee$ & $\because \cdot 109 \mathrm{~V}$ & $9.11 \leq 00$ & $\because \cdots r r$ \\
\hline$V r$ & 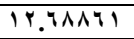 & $\because \cdot r q \leq V$ & $11.0 .74 \%$ & $\because \cdots \wedge \wedge$ \\
\hline$V \leq$ & r.IV90 & $\because \cdots 1 \vee 0$ & $r .0 \wedge I V \wedge$ & $\because \cdots \wedge$ \\
\hline Vo & $.90 \vee 0 \leq$ & $\because \cdots v \leq$ & $\cdot \wedge V \cdot I r$ & $\because \cdots 9$ \\
\hline VI & r..0rrqr & $\cdot r \mid \wedge / r$ & $1 \leq . \vee \wedge .9 r$ & . . rVr \\
\hline$V V$ & $17.99 Y \wedge \leq$ & $\because .5710$ & 9.YOY. & . P YVY \\
\hline$\vee \wedge$ & $1 . \cdot r \leqslant \Lambda r$ & 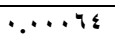 & $\cdot .9 \leq Y Y 1$ & $\because \cdots \wedge$ \\
\hline Vq & 1.1YKYE & $\because \cdots \circ V$ & 1.1771 & $\because \cdots v$ \\
\hline$\Lambda$. & $1.7 . r \vee q$ & .00 .9 & I.VYYAT & 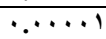 \\
\hline$\Lambda 1$ &. $.890 \% q$ & $\because \cdots 1.9$ & $\cdot . \vee \wedge \wedge \vee$ & $\because \cdots 1$ \\
\hline Ar & $1 . r \leq r V T$ & $\because \cdots v r$ & 1.47199 & $\ldots \ldots \varepsilon$ \\
\hline 83 &.$\wedge 970$. & $\because \cdots 109$ & •.AM. I & $\because \cdots 1 \leq$ \\
\hline$\Lambda \leq$ & $\cdot . \wedge \leq 7 \vee \wedge$ & $\because \cdots 1 \leq \Lambda$ &. $.4 \wedge \neg 1 \%$ & $\because \cdots$ \\
\hline No & $\Lambda .0 \leq 79 \wedge$ & $\because \cdots \wedge, \varepsilon$ & $r \cdot .007 V \wedge$ & . SqYr \\
\hline$\Lambda 7$ & $\cdot V 7 \leq r q$ & $\because \cdots 1$ ro & $\because V \ldots r r$ & $\because \cdots \cdots 9$ \\
\hline$\Lambda V$ & •.ATYVV & $\because \cdots 9 r$ & .VOYY. & $\because \cdots 9$ \\
\hline$\wedge \wedge$ & $r . V \leq . \wedge 0$ & $\because \cdots r o$ & r.rumar & $\because \cdots r \leq$ \\
\hline 19 & $\cdot . \vee \wedge q \vee q$ & $\because \cdots 11 \leq$ & .VITYO & ...1. \\
\hline 9. & $1.9 .70 \mathrm{~V}$ & ..rr. & Ir.9YYIA &. $.191 \mathrm{VA}$ \\
\hline 91 & $1 \leqslant . V Y \leqslant Y$ & $\because \bullet \vee \vee \wedge \wedge$ & $V .9 \leq 71 \%$ & ...YYr \\
\hline QY & $\varepsilon . \leqslant \vee \vee \backslash \wedge$ & $\because \cdot 1$, vo & $r .57091$ & $\because \cdots \leq Y$ \\
\hline 94 & $1.591 \leq 7$ & . ROM & $1.1184 r$ & $\because \cdots 10$ \\
\hline $9 \leq$ & YY.VV.rs & $\because+\leqslant V Y 0$ & Ir.AYYAT & $\because \cdots \wedge \leq$ \\
\hline 90 & Y.\{^०५. & $\because \cdots 99$ & 1.9 .974 & $\because \cdots r V$ \\
\hline 97 & $1 . Y \cdot \leq 00$ & $\because \cdots 7 r$ & $1 . \cdot \leq 191$ & $\because \cdots, 0$ \\
\hline $9 V$ &. $.90 \wedge \ldots$ & $\because \cdots \wedge 1$ & $\cdot . \wedge \leq 90$. & $\because \cdots$ \\
\hline 91 & •.V94.. & $\because \cdots 1 \cdot 1$ & $\cdot V 1 \leq r 0$ & $\because \cdots \wedge$ \\
\hline 99 &. $.99 \vee 11$ & $\because \cdots \leq 4$ & $1 . r \cdot r \wedge 1$ & $\because \cdots \cdots$ \\
\hline
\end{tabular}




\begin{tabular}{|c|c|c|c|c|}
\hline $1 \ldots$ & $. .9 \leqslant 4.7$ & $\because \cdots \wedge 1$ & Y.1 1 Vर० & . raro \\
\hline 1.1 & . .qVRr। & $\because \cdots Y r$ & $.971 \times 9$ & $\because \cdots \wedge$ \\
\hline $1 . r$ &.$V 0 V 10$ & $\because \cdots I r$ & $\cdot . \neg \wedge 9 \vee \wedge$ & $\because \cdots 9$ \\
\hline $1 . r$ & $\cdot V \leq \cdot \leq \varepsilon$ & $\because \cdots 11 r$ & $.7 \leq 00 \leq$ & $\because \cdots v$ \\
\hline 1.5 & $\cdot V \leqslant V Y$. & $\because \cdots 11 r$ & $\cdot . \neg \vee \diamond \vee \wedge$ & $\because \cdots 9$ \\
\hline 1.0 & .991 .71 & $\because \cdots v \varepsilon$ & $\cdot . \wedge 9 \cdot 19$ & $\because \cdots 9$ \\
\hline 1.7 & $\cdot . \wedge \leq \leqslant \vee 0$ & $\because \cdots 9 \wedge$ &. .117 .1 & $\because \cdots$ \\
\hline $1 . V$ & . VTMIE & $\because \cdots / r$ & 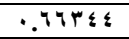 & $\because \cdots 9$ \\
\hline 1.1 & I.Y.rok & $\cdot \cdot|r \wedge|$ & $\cdot \wedge 9 / Y q$ & . PYYIY \\
\hline 1.9 & $1.7 V \leq 0 \leq$ & $\because \cdot 1 \cdot M 1$ & .VYYV. & $\because \cdots 1 \wedge$ \\
\hline 11. & $.0 \wedge$ TYY & $\because \cdot 1.91$ & $\cdot r 9 \cdot 11$ & $\because \cdots v$ \\
\hline 111 & 1.7 .101 & $\because \cdots r$ & $1.10 \ldots 1$ & $\because \cdots 1$ \\
\hline $11 Y$ & . VOY.. & $\because \cdots 11 r$ & $\cdot . T V \leqslant r 0$ & $\because \cdots \wedge$ \\
\hline $11 \%$ & . VqAr. & $\because \cdots 11 \wedge$ & •VYVTY & $\because \cdots 11$ \\
\hline 118 & $\because V \vee 0, Y$ & $\because \cdots \mid r \Lambda$ & $\cdot V Y \cdot \Lambda \cdot$ & $\because \cdots / r$ \\
\hline 110 & $1.1 Y V I \leq$ & $\because \cdots 7 \varepsilon$ & $11.1 r \leq \leqslant 1$ &. .10819 \\
\hline 117 &. $.779 \vee Y$ & $\because \cdots 1$ ro & $\cdot . \neg \leqslant \wedge r$ & $\because \cdots 9$ \\
\hline 118 & Y.VVOYY & $\because \cdots \cdots$ & $\cdot . \neg \leqslant \wedge r$ & $\because \cdots 9$ \\
\hline 111 & 1.5471. & $\because \cdots 74$ & 1.rrork & $\because \cdots$ \\
\hline 119 & $. \wedge r \leqslant q q$ & $\because \cdots 9 v$ & $\cdot V r \cdot Y \leq$ & $\because \cdots \cdots$ \\
\hline IY. & $0.0 Y 11 \leq$ & $\because \cdots \vee q$ & $0.49 \leq 71$ & $\because \cdots 19$ \\
\hline$|Y|$ &. .19 .90 & $\because \cdots / r$ & $\because \vee 1 \wedge 97$ & $\because \cdots 11$ \\
\hline IYY & $1.114 \leq 7$ & $\because \cdots v$. & $1 . .511 V$ & $\because \cdots 9$ \\
\hline IrT & $\leq .17 Y \leq 7$ & $\because \cdots r$ & $1 . . \leq 11 V$ & $\because \cdots 9$ \\
\hline IYs & ؛.IVAษ & $\because \cdots \cdots$ & $5.194 \wedge r$ & $\because \cdots 1 V$ \\
\hline 1ro & $\Lambda . \leqslant \wedge 901$ & $\because \cdots \wedge 1$ & r.rlorq & $\because \cdot 7 \wedge \cdot V$ \\
\hline $1 Y 7$ & $1 . r \leq 0 \vee V$ & $\because \cdots \leq r$ & $1 . Y \leq \vee \wedge \bar{T}$ & $\because \cdots v$ \\
\hline IYV & $17 . \wedge 99 \mathrm{rr}$ & $\because \cdots V Y \leq$ & $1 . Y \leq \vee \wedge 7$ & $\because \cdots v$ \\
\hline $1 Y \Lambda$ & r.sIAY. & $\because \cdot 1 \wedge 9 r$ & $r . \leqslant 90 . V$ & .... rq \\
\hline $1 \times 9$ & $\cdot . \wedge \neg \leqslant 0 \wedge$ & $\because \cdots 11 r$ & 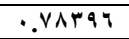 & $\because \cdots 1 r$ \\
\hline $1 \%$ & 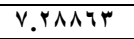 & $\because \cdot 1 r \leq 9$ & $\vee . \wedge \circ \vee \cdot q$ & $\because \cdots \cdots$ \\
\hline 141 & $1 . .774$ & $\because \cdots \leq \leqslant \wedge$ & $1.17 \leq 0$. & 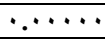 \\
\hline IrY & -.VYqYY & 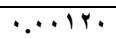 & $. .74 . \leqslant Y$ & $\because \cdots 1$. \\
\hline Irr & $\because \wedge \wedge \mu_{1}$ & $\because \cdots \leq \wedge 1$ & V.รัง V & $\because \cdot \leqslant \vee \wedge$. \\
\hline $1 T \varepsilon$ & V.MIIAY & $\cdot \ldots \ldots$ & $V .9 .1 Y q$ & ...VVr \\
\hline 140 & N.MIY०q & $\because \cdots 11 \mathrm{~V}$ & $\Lambda . \vee 9 \leq 01$ & $\because \cdots 11 \mathrm{~V}$ \\
\hline 149 & $\Lambda . Y \backslash 7 \leqslant \Lambda$ & $\because \cdots \leq V Y$ & V.7.7Vq & $\because \cdots+1$ \\
\hline IrV & I. KYYq & $\because \cdots v$. & $.9479 \wedge$ & ...1. \\
\hline $1 \mathrm{NA}$ & . .79 & $\because \cdots 171$ & $.90 Y 19$ & $\because \ldots 9$ \\
\hline 149 & I.MYYIT & $\because \cdots \circ V$ & $1 . Y \cdot \leq \wedge 1$ & $\because \cdots v$ \\
\hline $1 \leq$. & $\cdot 9 V \leq r 0$ & $\because \cdots v V$ & D.VVYAO & $\because . \mathrm{YqIV}$ \\
\hline $1 \leq 1$ & $1.101 Y Y$ & $\because \cdots, 0$ & $1 . \cdot \leq 0 \wedge r$ & $\because \cdots \wedge$ \\
\hline $1 \leq Y$ & $\cdot . \wedge \wedge \vee \backslash 0$ & $\because \cdots \wedge \varepsilon$ & $\cdot \Lambda \cdot r \circ \leq$ & $\ldots \ldots 9$ \\
\hline $1 \leqslant r$ & $.009 \wedge 4$ & $\because \cdot 11 \mathrm{VV}$ & .00170 & 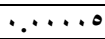 \\
\hline $1 \leq \leq$ & .9 .97V & $\because \cdots \vee v$ & $\cdot$ ANrII & $\because \cdots \wedge$ \\
\hline $1 \leqslant 0$ & $\cdot . \wedge \leqslant \wedge \vee q$ & $\because \cdots r r$ & I..PYY & $\because \cdots v$ \\
\hline $1 \leq 7$ & . АTY४। & $\because \cdots 9 v$ & .vorav & $\because \cdots 1$ \\
\hline $1 \leqslant V$ & $V_{.} . \Delta V \leq$. & $\cdot \cdot 1 \leq 1 r$ & V.PVRYq &.$\ldots 17$ \\
\hline $1 \leqslant \Lambda$ & $1.7 r \leqslant v 0$ & $\because \cdot 19 \pi \leq$ & $\cdot \Delta r \cdot r l$ & $\because \cdot r 1 \cdot r$ \\
\hline $1 \leq 9$ & 0.049. & $\because \cdots r \wedge$ & $0 . Y \wedge \neg . r$ & $\because \ldots$ \\
\hline 10. & $0.17 \leqslant 0$. & $\because \cdots 1$ rq & $0.1 \leq 00 \leq$ & $\cdot \cdots \cdot r$ \\
\hline
\end{tabular}


Figure (3.9) Serbert before $(n=150)$

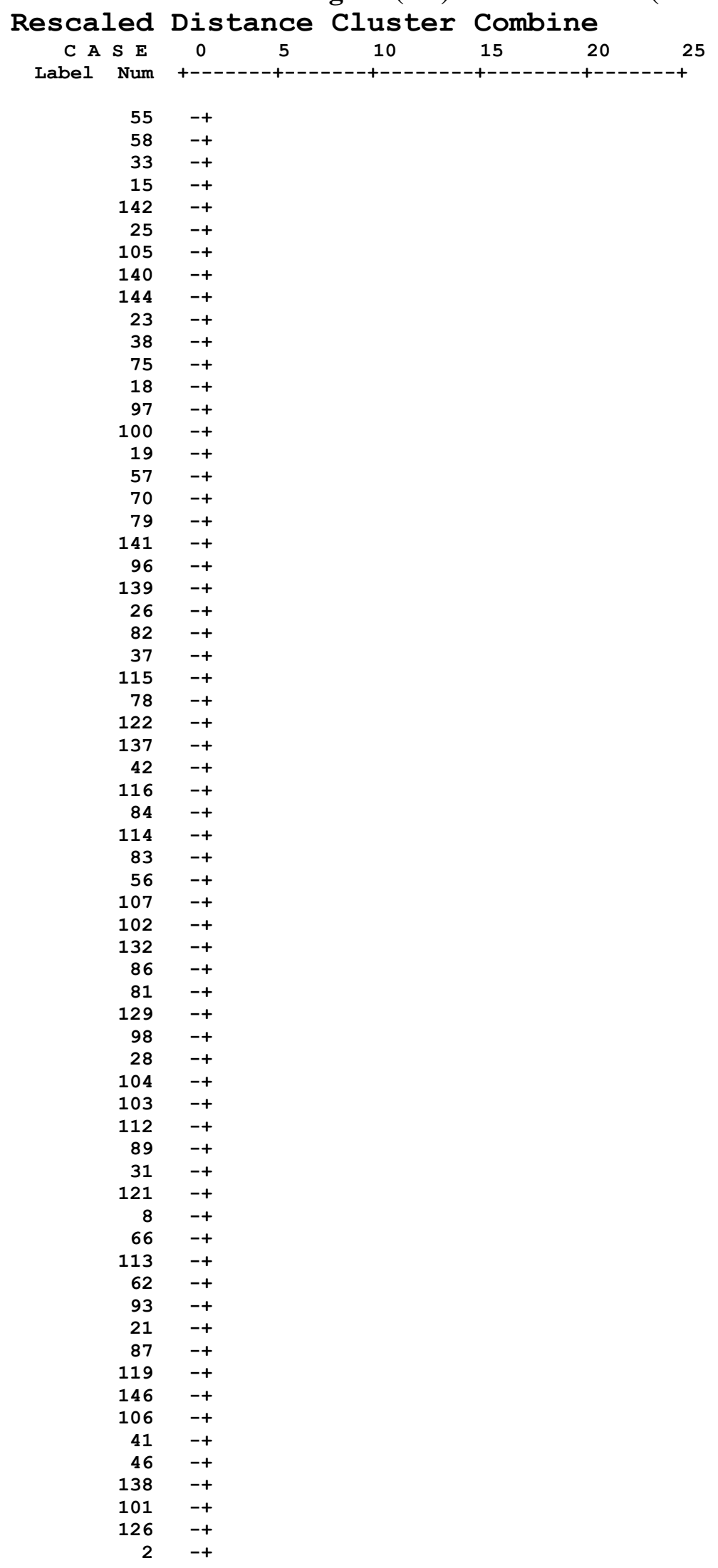




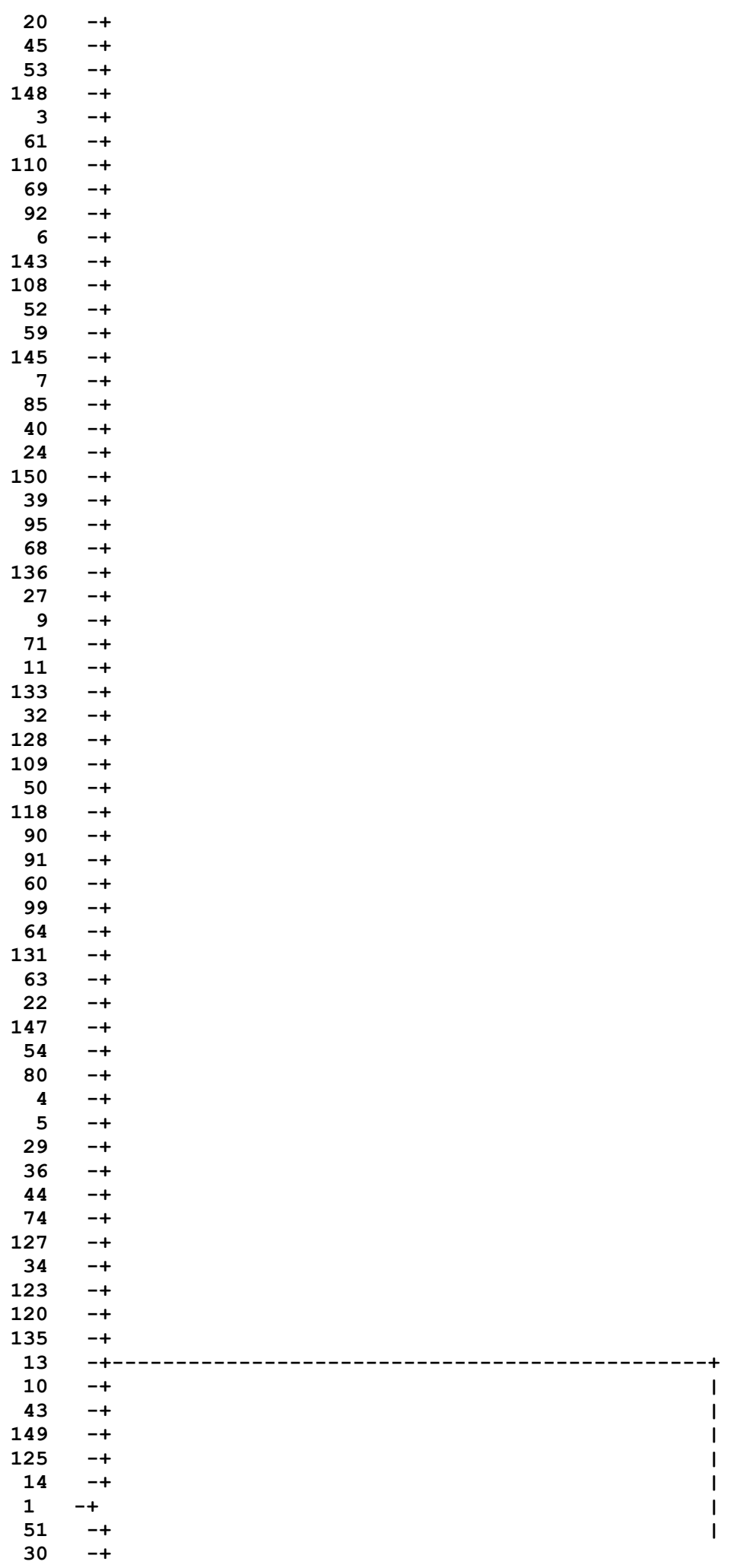




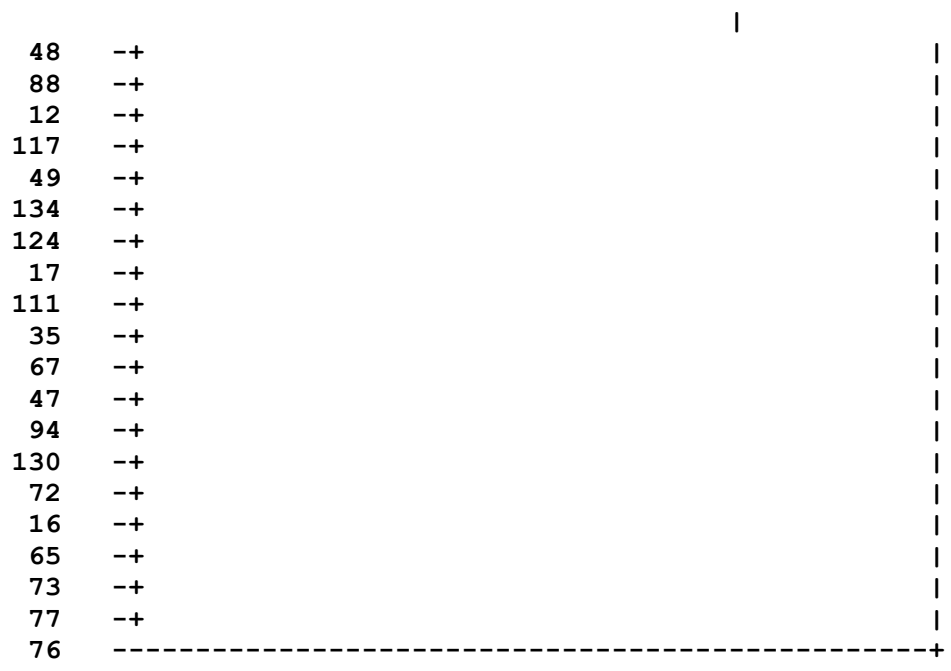

Figure (3.10) Adnan before ( $n=150)$

Rescaled Distance Cluster Combine

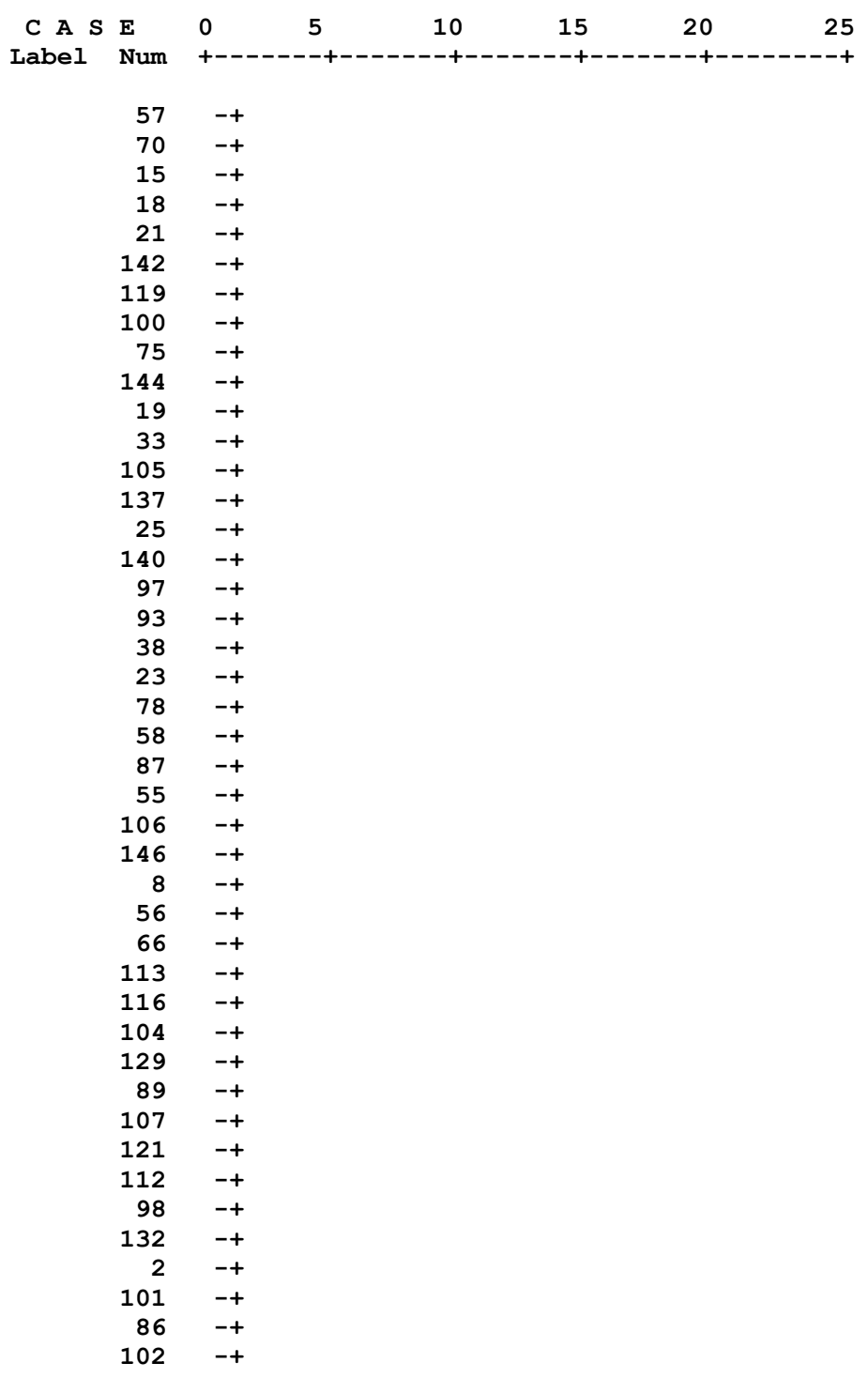




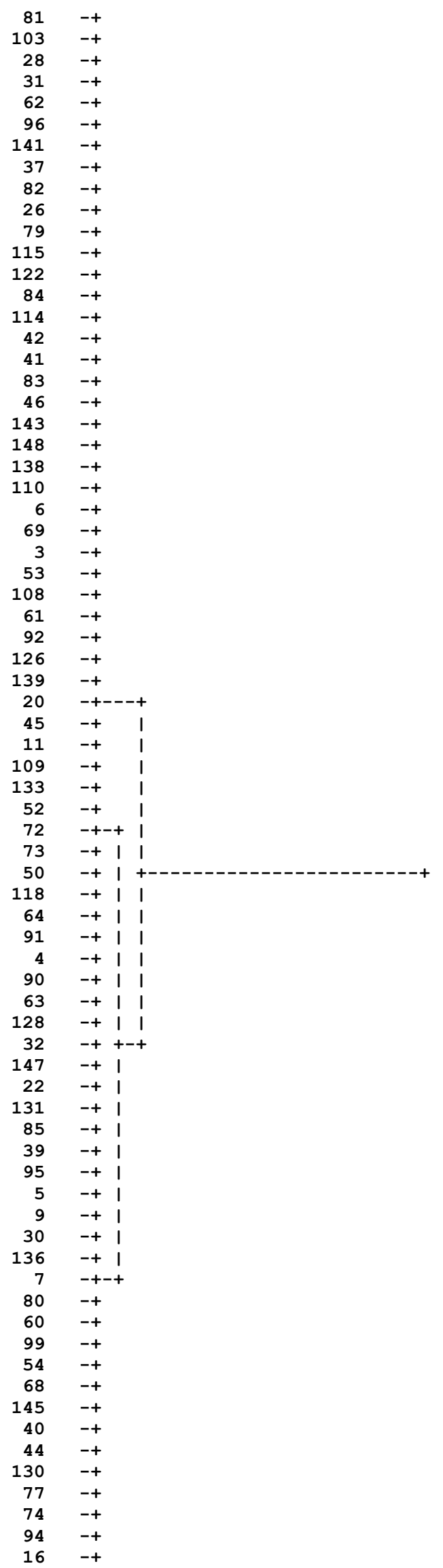




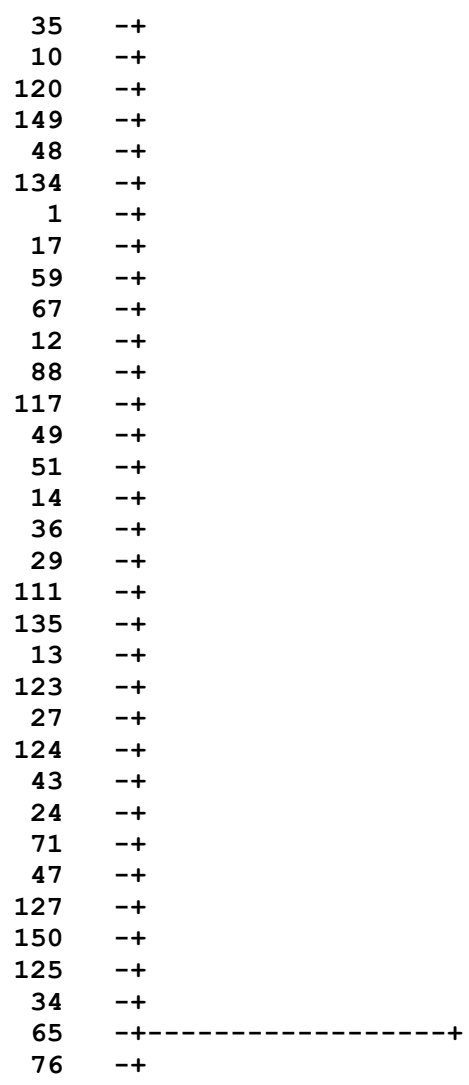

Figure (3.11) Serbert after $(n=150)$

Rescaled Distance Cluster Combine

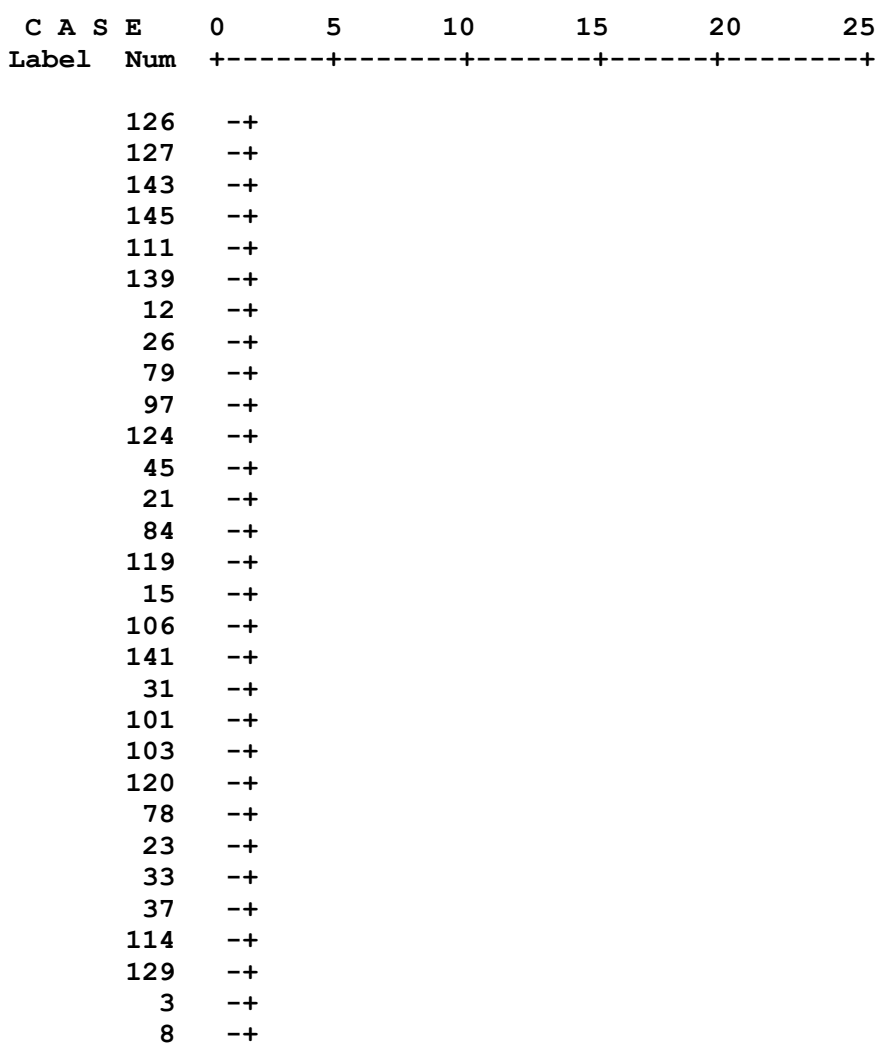




$\begin{aligned} 81 & -+ \\ 132 & -+ \\ 53 & -+ \\ 138 & -+ \\ 6 & -+ \\ 66 & -+ \\ 67 & -+ \\ 49 & -+ \\ 52 & -+ \\ 34 & -+ \\ 69 & -+ \\ 146 & -+ \\ 107 & -+ \\ 46 & -+ \\ 116 & -+ \\ 117 & -+ \\ 86 & -+ \\ 41 & -+ \\ 74 & -+ \\ 104 & -+ \\ 137 & -+ \\ 18 & -+ \\ 102 & -+ \\ 147 & -+ \\ 87 & -+ \\ 142 & -+ \\ 14 & -+ \\ 144 & -+ \\ 118 & -+ \\ 38 & -+ \\ 112 & -+ \\ 39 & -+ \\ 98 & -+ \\ 56 & -+ \\ 75 & -+ \\ 28 & -+ \\ 57 & -+ \\ 25 & -+ \\ 105 & -+ \\ 122 & -+ \\ 123 & -+ \\ 19 & -+ \\ 62 & -+ \\ 121 & -+ \\ 55 & -+ \\ 89 & -+ \\ 58 & -+ \\ 128 & -+ \\ 113 & -+ \\ 9 & -+ \\ 7 & -+ \\ 11 & -+ \\ 83 & -+ \\ 149 & -+ \\ 16 & -+ \\ 42 & -+ \\ 68 & -+ \\ 92 & -+ \\ 115 & -+ \\ 36 & -+ \\ 32 & -+ \\ 99 & -+ \\ 40 & -+ \\ 47 & -+ \\ 63 & -+ \\ 64 & -+ \\ 210 & -+ \\ 96 & -+ \\ 96 & -+\end{aligned}$




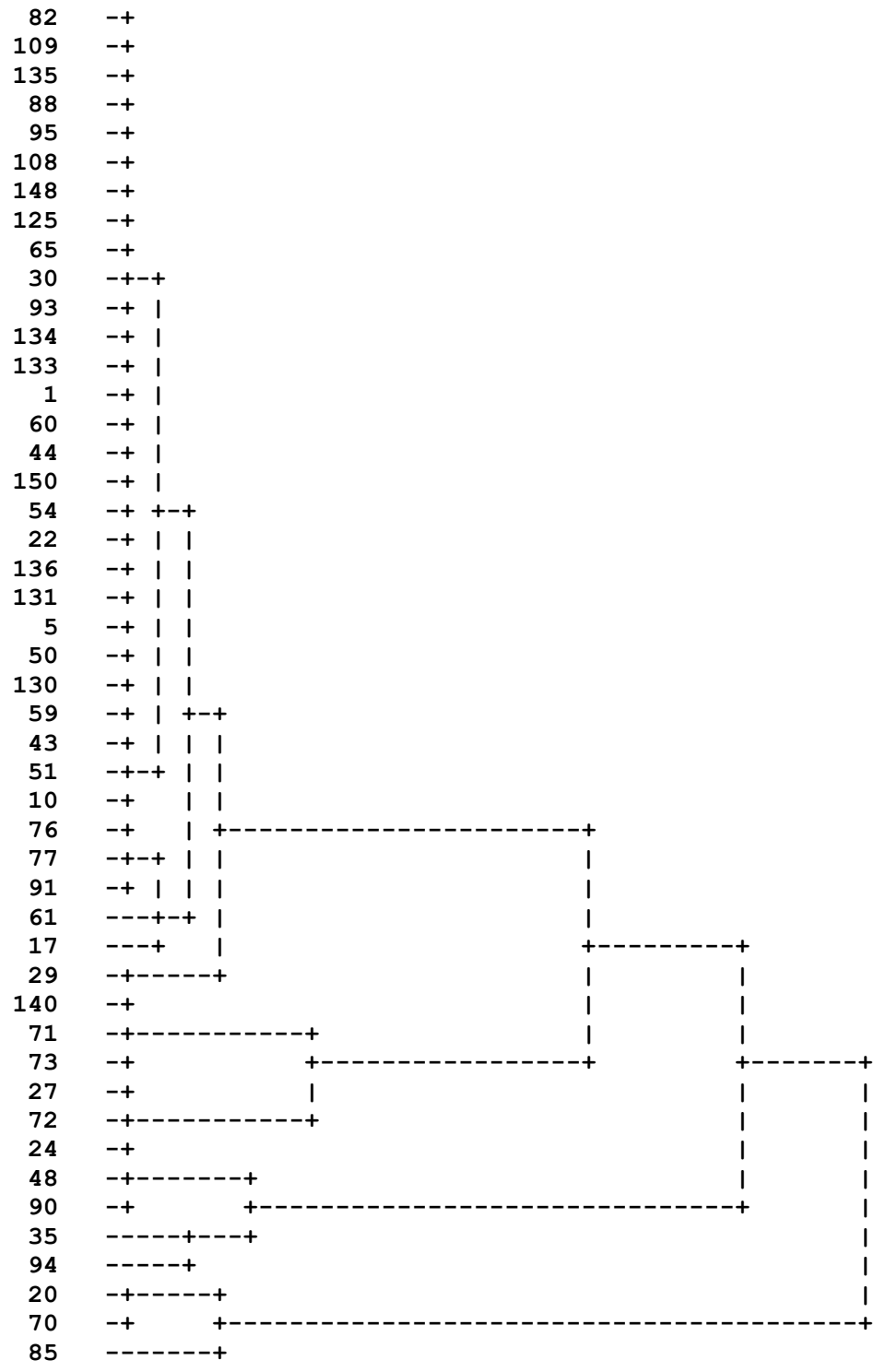


rv

Figure (3.12) Adnan after $(n=150)$

Rescaled Distance Cluster Combine

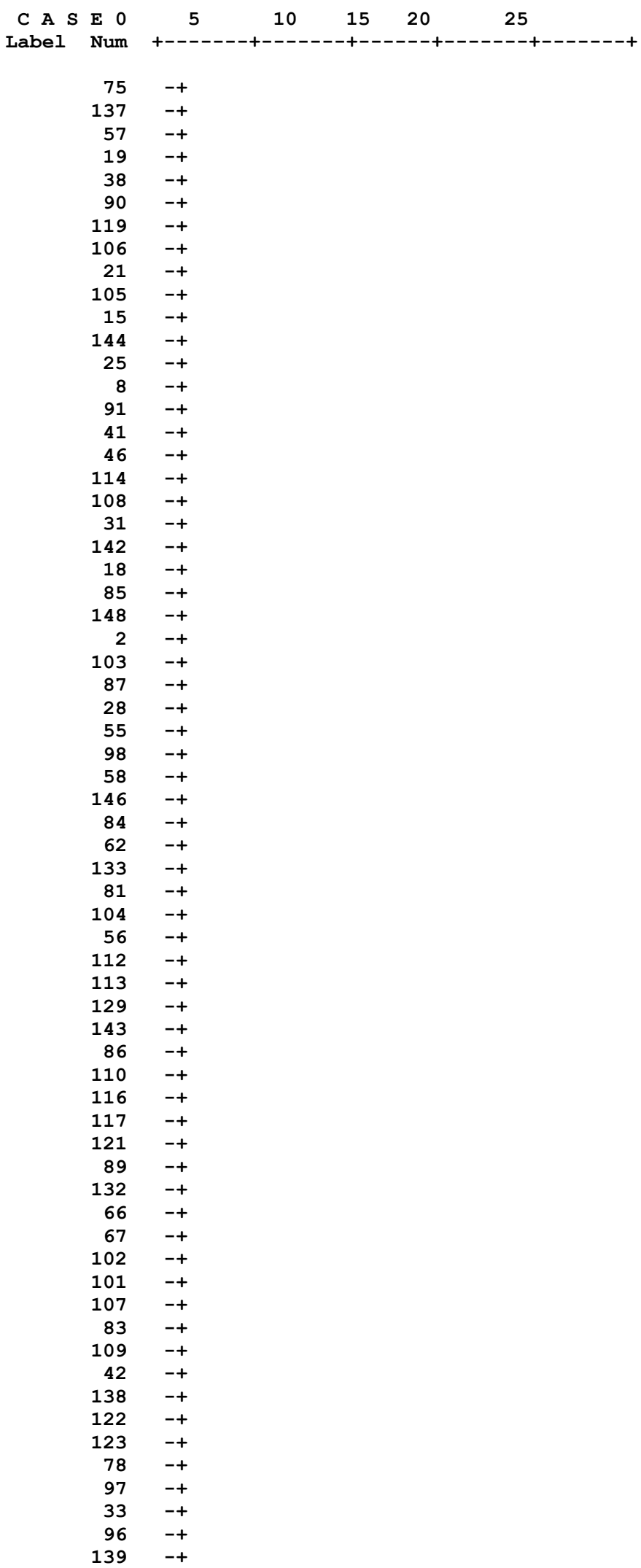




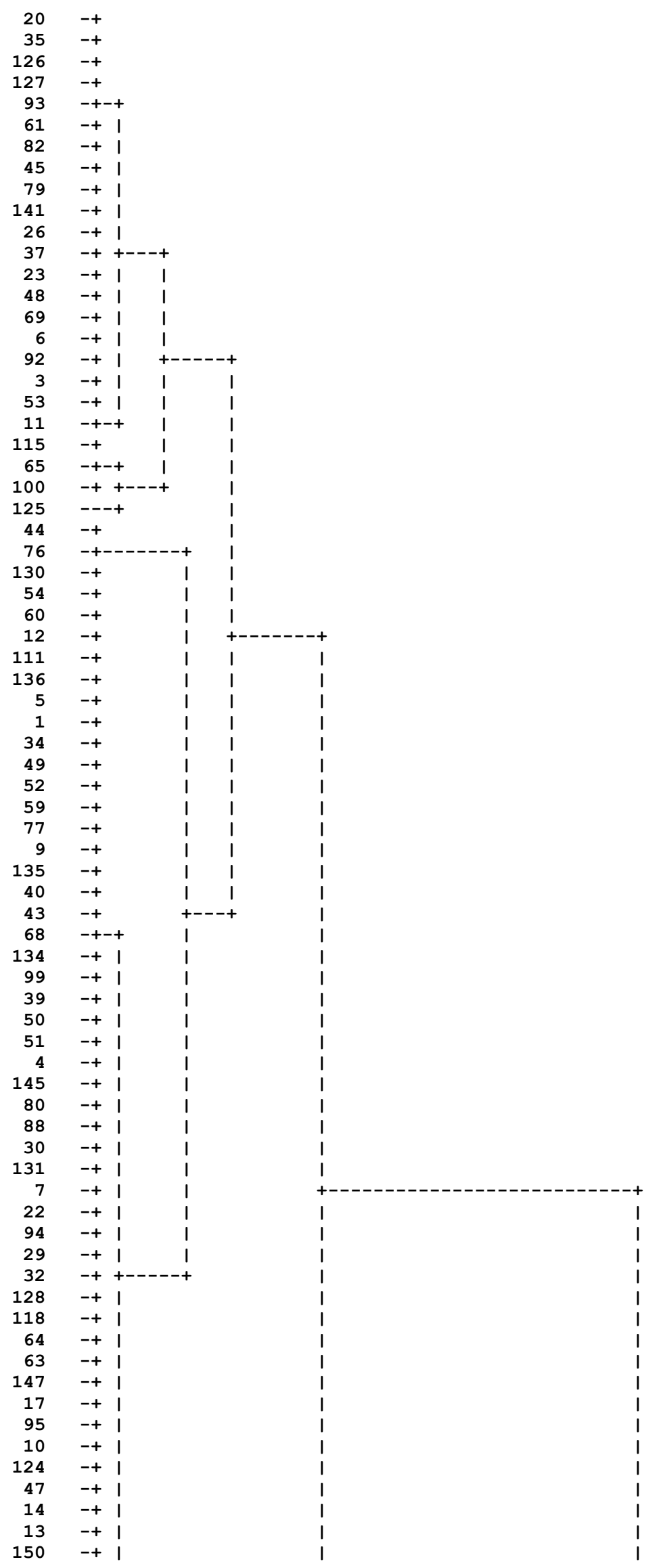




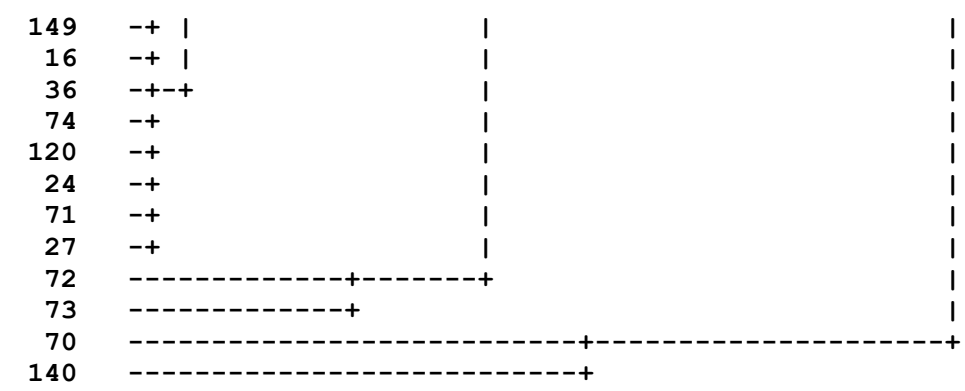

Table (3.9) below shows the summary for the methods, where they are all compared with Mahalanobis distance $\left(\chi_{(145,0.05)}^{2}=67.2\right)$, and Cook distance $\left(F_{(5,45,0.05)}=2.21\right)$. Both of them can not detect any outliers in the two cases. Outliers are detected by Serbert and Adnan, depending on (ch) value . Differences are found between them for before and after cases .

Mahalanobis and Cook have masking for all the adding outliers ; but Serbert has in the No. $(10,65,100,108,115,125,133,148)$; and Adnan has in the No. $(20,35,48,65,85,90,100,108,115,125,133,148)$

Mahalanobis and Cook didn't have swamping ; but Serbert has in the No. $(24,27,71,72,73,94) \quad ; \quad$ and Adnan has in the No.(13,14,16,17,24,27,36,47,63,64,71,72,73,74,95,118,120,124,128,147,149,150) .

Mahalanobis, Cook and Serbert have (0.3) Std. Error Est. before case , and Serbert has (2.51) after case, which are less than others . 
Table (3.9) Summary $(n=150)$

\begin{tabular}{|c|c|c|c|c|c|c|}
\hline case & method & ch & outliers & masking & swamping & $\begin{array}{l}\text { Std. } \\
\text { Error } \\
\text { Est. }\end{array}$ \\
\hline \multirow{5}{*}{ Before } & none & - & - & - & - & 0.3 \\
\hline & Mah. & - & none & - & - & 0.3 \\
\hline & Cook & - & none & - & - & 0.3 \\
\hline & Serbert & 12.5 & $\begin{array}{l}1,10,12,13,14,16,17, \\
30,35,43,47,48,49,51,65,67,7 \\
2,73,76,77,88,94,111,117,124 \\
, 125,130,134,149)\end{array}$ & - & - & 0.3 \\
\hline & Adnan & 3.6 & $\begin{array}{l}(1,10,12,13,14,16,17, \\
24,27,29,34,35,36,40,43,44,4 \\
7,48,49,51,54,59,60,65,67,68, \\
71,74,77,80,88,94,99,111, \\
117,120,123,124,125,127,130 \\
, 134,135,145,149,150)\end{array}$ & - & - & 0.33 \\
\hline \multirow{5}{*}{ After } & none & - & - & - & - & 3.21 \\
\hline & Mah. & - & - & $\begin{array}{l}10,20,35, \\
48,65,70, \\
85,90,100,108 \\
, 115,125,133, \\
140,148 \\
\end{array}$ & - & 3.21 \\
\hline & Cook & - & - & $\begin{array}{l}10,20,35, \\
48,65,70, \\
85,90,100,108 \\
, 115,125,133, \\
140,148 \\
\end{array}$ & - & 3.21 \\
\hline & Serbert & 5 & $\begin{array}{l}(20,24,27,35,48,70,71,72,73,8 \\
5,90,94,140)\end{array}$ & $\begin{array}{l}10,65,100,108 \\
, 115,125,133, \\
148\end{array}$ & $\begin{array}{l}\text { 24,27,71, } \\
72,73,94\end{array}$ & 2.51 \\
\hline & Adnan & 4.3 & $\begin{array}{l}(10,13,14,16,17,24,27,36,47,6 \\
3,64,70,71,72,73,74,95,118,1 \\
20,124,128,140,147,149,150)\end{array}$ & $\begin{array}{l}20,35,48 \\
65,85,90 \\
100,108 \\
115,125 \\
133,148\end{array}$ & $\begin{array}{c}13,14,16, \\
17,24,27, \\
36,47,63, \\
64,71,72 \\
73,74,95 \\
118,120 \\
124,128 \\
147,149,150\end{array}$ & 3.05 \\
\hline
\end{tabular}

4. Conclusions :

- Mahalanobis and Cook Methods could not detect any outlier for all cases, although many of outliers are inserted in the observations. This is because both of them depend on detecting a single outlier, and if the outliers are grouping, they may have detected them .

- When (n=25), Adnan's method has the smallest (Std. Error Est.) for before case . It has the same results of Serbert method's after adding the outliers and both of them reduced (Std. Error Est.) .

- When $(\mathbf{n}=50)$, Serbert method's has the smallest (Std. Error Est.), masking and swamping •

- When (n=150), Serbert method's has the smallest (Std. Error Est.) , masking and swamping . 


\section{References :}

- Adnan,R.; Mohamad,M.N. and Setan,H. (2003) "Multiple Outliers Detection Procedures in Linear Regression" , Matematika ,2003,Jilid 19 ,bil. 1,hlm.(2945) .

- Asikgil, Baris and Erar, Aydin (2009) "Research Into Multiple Outliers in Linear Regression Analysis" . Journal of mathematics and Statistics Volume 38 (2) , (185-198) .

- Barnett, V. and Lewis, T. (1994) "Outliers in Statistical Data ", $3^{\text {rd }}$ ed. John Wiley .

- Chen, Colin . (2003) . "Robust Regression and Outlier Detection with the ROBUSTREG Procedure" . http://www2.sas.com/proceedings/sugi27/p265-27.pdf

- Cook, R.D. (1979) "Influential Observations in Linear Regression" „JASA. 74,(169174) .

- Draper, N.R. \& John, J.A.(1981) "Influential Observations and Outliers in Regression " Technometrics ,23, (21-26) .

- Elashoff, J.D. (1972) "A Model for Quadratic Outliers in Linear Regression " ,JASA,67,(478-485) .

- Gal, I. B. (2005)" Outlier detection" http://www.eng.tau.ac.il/ bengal/outlier.pdf

- Georgiev, T.B. (2008) " An introduction to the method of the robust regression of Rousseeuw ", Astrophys. Invest 10,(93-116) .

- Karpinski, A. (2007) " Simple Linear Regression " .

- McCane, B. (2009) "Mahalanobis Distance" • http://www.mailarchive.com/morphmet @morphometrics.org/msg01466/mahal.pdf

McLachlan, G.J. (1999) "Mahalanobis Distance" . http://www.ias.ac.in/resonance/June1999/pdf/June1999p20-26.pdf

- Mishra, SK. (2008) "A New Method of Robust Linear Regression Analysis : Some Monte Carlo Experiments". http://papers.ssrn.com/sol3/papers.cfm?abstract_id=1155135

Pena, D. \& Yohai, V. (1999)" A Fast Procedure for Outlier Diagnostics in Large Regression Problems " JASA ,94,(434-445) .

- Rousseeuw, P.J.(1984)" Least Median of Squares Regression " , JASA . 79,(871880) .

- Serbert, D.M. (1998) " A clustering algorithm for identifying multiple outliers in linear regression " , Computational Statistics \& Data Analysis , 27,(461-484) . 